\title{
Enhancing the ionic conductivity of solid state electrolytes by oxide glass doping
}

Yue Zhou

Follow this and additional works at: https://researchrepository.wvu.edu/etd

\section{Recommended Citation}

Zhou, Yue, "Enhancing the ionic conductivity of solid state electrolytes by oxide glass doping" (2016). Graduate Theses, Dissertations, and Problem Reports. 7051.

https://researchrepository.wvu.edu/etd/7051

This Thesis is protected by copyright and/or related rights. It has been brought to you by the The Research Repository @ WVU with permission from the rights-holder(s). You are free to use this Thesis in any way that is permitted by the copyright and related rights legislation that applies to your use. For other uses you must obtain permission from the rights-holder(s) directly, unless additional rights are indicated by a Creative Commons license in the record and/ or on the work itself. This Thesis has been accepted for inclusion in WVU Graduate Theses, Dissertations, and Problem Reports collection by an authorized administrator of The Research Repository @ WVU. For more information, please contact researchrepository@mail.wvu.edu. 


\title{
Enhancing the ionic conductivity of solid state electrolytes by oxide glass doping
}

\author{
Yue Zhou \\ Thesis submitted to \\ Benjamin M. Statler College of Engineering and Mineral Resources \\ at West Virginia University \\ in partial fulfillment of the requirements \\ for the degree of \\ Master \\ In \\ Mechanical Engineering \\ Xingbo Liu, Ph.D., Chairperson and Advisor \\ Xueyan Song, Ph.D. \\ Bingyun Li, Ph.D.
}

Department of Mechanical and Aerospace Engineering

Morgantown, West Virginia

2016

Keywords: Lithium, ion conductivity, LLTO, oxide glass doping Copyright 2016 [Yue Zhou] 


\begin{abstract}
As the increasing use of lithium ion secondary batteries in practical application, solid state electrolyte attracted lots of attention since it's a crucial part for the lithium ion secondary batteries. Compare with liquid electrolyte, the advantage of solid state electrolyte covering no electron leakage, safety, chemical and thermal stability, high energy density and so on. However, one disadvantage of inorganic solid state electrolyte is the low ionic conductivity which is primarily due to its low grain boundary conductivity. Among all of the solid state electrolyte candidates for lithium battery applications, the perovskite structure lanthanum lithium titanate ( $\mathrm{La}_{2 / 3-\mathrm{x}} \square_{1 / 3}$ ${ }_{2 x} \mathrm{Li}_{3 \mathrm{x}} \mathrm{TiO}_{3}$ ) has the best lithium ionic conductivity, which can reach $10^{-3} \mathrm{~S} / \mathrm{cm}$ in the grain bulk, but typically only $10^{-6} \mathrm{~S} / \mathrm{cm}$ at the grain boundary.

It has been found that the addition of amorphous phases doped into the LLTO grain boundary can significantly improve its overall conductivity. Several candidates for use as a suitable, high conducting amorphous dopant with the best properties were considered. The sulfide glass system is widely recognized as being the highest lithium ion conducting amorphous system. However, the difficulties associated with its fabrication and instability in air along with possible reactions with oxide materials are major drawbacks to its application as a dopant in the LLTO system. These are not problems found in the oxide glass system and so we looked at several oxide glass formers. Previously, people introduced boron trioxide, silica and alumina as single composition amorphous phase dopants into LLTO in an effort to improve its grain boundary conductivity. To obtain further enhancements to the grain boundary ionic conductivity, composite composition oxide glasses were investigated. As it turns out, some of these systems can reach the ionic conductivity of LLTO grain bulk.
\end{abstract}


In the present work, we used the melt quench method to make different compositions of the $0.4 \mathrm{Li}_{2} \mathrm{O}+0.25 \mathrm{~B}_{2} \mathrm{O}_{3}+(0.35-\mathrm{x}) \mathrm{SiO}_{2}+\mathrm{xAl}_{2} \mathrm{O}_{3}$ glass system and doped these into the LLTO grain boundary to enhance its ionic conductivity. $\mathrm{SiO}_{2}$ is generally used as a base glass former. Substitution of Si by $\mathrm{Al}$ is believed to decrease the activation energy of the glass, and furthermore, $\mathrm{B}_{2} \mathrm{O}_{3}$ and $\mathrm{Al}_{2} \mathrm{O}_{3}$ can be treated as a network former of alkali glass. Our result shows that, at room temperature, the best conductivity can reach $3.4 \times 10^{-4} \mathrm{~S} / \mathrm{cm}$ at the grain boundary. The amount of $\mathrm{Al}_{2} \mathrm{O}_{3}$ was found to play a crucial role in the conductivity of this glass system. To gain further understanding, we examined the morphology of the LLTO/oxide glass pellet cross section and tested the density of these pellets to confirm the amount of oxide glass necessary to fully fill space at the LLTO grain boundary. 


\section{Acknowledgments}

First of all, please let me express my earnest appreciation to Dr. Xingbo Liu. Thanks him to give me the chance to study and work with as a master student. Dr. Liu always keep the step on the leading edge of science and technologies in lithium battery. In our every work, he always gives me the right research orientation and let me exert freely on experiment. I was benefit a lot from Dr. Liu during the two-years master study.

I also want to thank Dr. Greg Collins and Dr. Wenyuan Li gave me the guide for the experiment. Dr. Collins taught me all the synthesis methods, glass fabricating process for our sample and repaired furnaces for me. Dr. Li taught the fundamental knowledge of electrochemical impedance and the EIS operation.

Finally, thanks Dr. Marcela Redigolo and Dr. Weiqiang Ding to training me all the experiment facilities conclude XRD, SEM, TEM and XPS. Thanks to other group members give the sincerely caring. 


\section{Table of Contents}

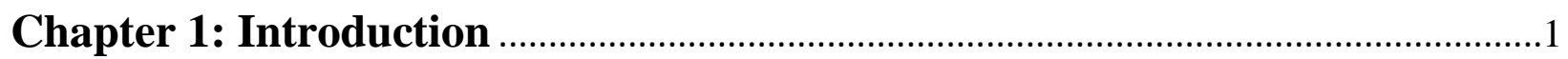

1.1 Solid state electrolytes in lithium-ion batteries.................................................................. 1

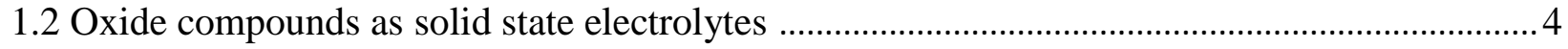

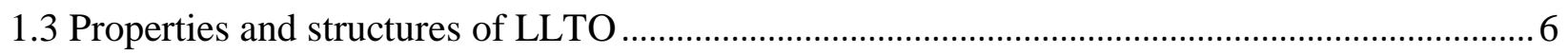

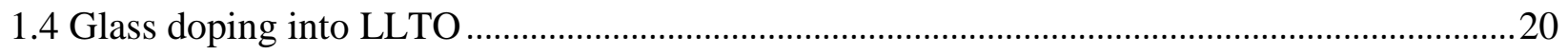

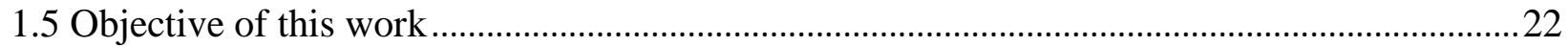

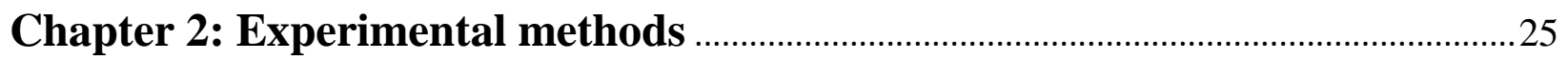

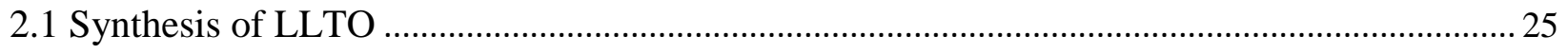

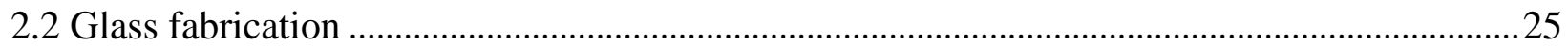

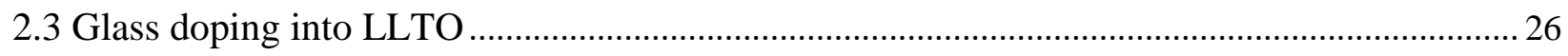

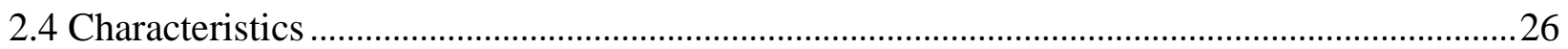

Chapter 3: Synthesis and characteristics of LLTO electrolyte ...............................27

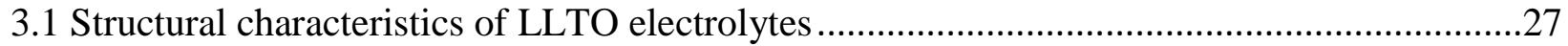

3.2 Examinations of lithium ions in LLTO electrolytes.................................................................28

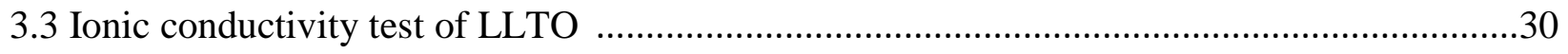

Chapter 4: Electrolytes doped with oxide glasses …............................................. 33

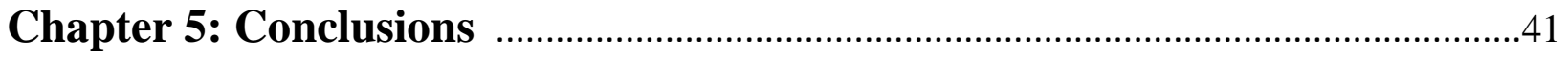

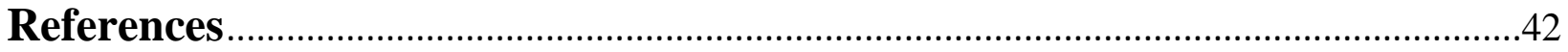




\section{Chapter 1: Introduction}

\subsection{Solid state electrolytes in lithium-ion batteries}

During the last two decades, the application of solid state electrolyte was very extensive which conclude high-energy ionic batteries, electrochromic system, supercapacitors and electrochemical sensors. Lead acid, nickel/cadmium (NiCd), nickel-metal hydride, and lithium ion batteries are the most commonly used rechargeable batteries. Lead acid batteries show both low gravimetric and volumetric energy densities. Nickel/cadmium ( $\mathrm{NiCd})$ and nickel-metal hybrid batteries have a much higher volumetric energy density, however, high self-discharge up to $10 \%$ per month is the main disadvantage of nickel/cadmium ( $\mathrm{NiCd}$ ) and nickel-metal hybrid batteries, so lithium ion batteries attract people's attention. Figure 1 indicates the volumetric energy density against gravimetric energy density for commonly used rechargeable batteries we mentioned above [1]. Furthermore, the performance of lead acid batteries, nickel/cadmium (NiCd) batteries and nickel metal hydride batteries can no longer satisfy the modern portable devices such as cell phone, laptop and so on. To achieve the requirements of the modern advanced portable devices and large scale energy storage devices, batteries we investigate need to proliferate higher energy generation, lighter weight and slimmer size. Safe and environmentally friendly is also necessary to consider about during the battery manufacture.

To achieve this complex combination of demands, during the last two decades, rechargeable lithium-based chemistries developed rapidly. Among all the elements that practically used in a battery system, lithium has the highest energy density because of the lowest electrochemical equivalent it has. Therefore, lithium batteries produce high gravimetric energy density and high volumetric energy density as shown in figure1. Compare to nickel/cadmium (NiCd) batteries and 
nickel metal hydride batteries, another advantage of lithium-based rechargeable batteries is the high unit cell voltage, the former two usually offer $1.5 \mathrm{~V}$, but the lithium based one usually can offer between 3.0-4.2V.

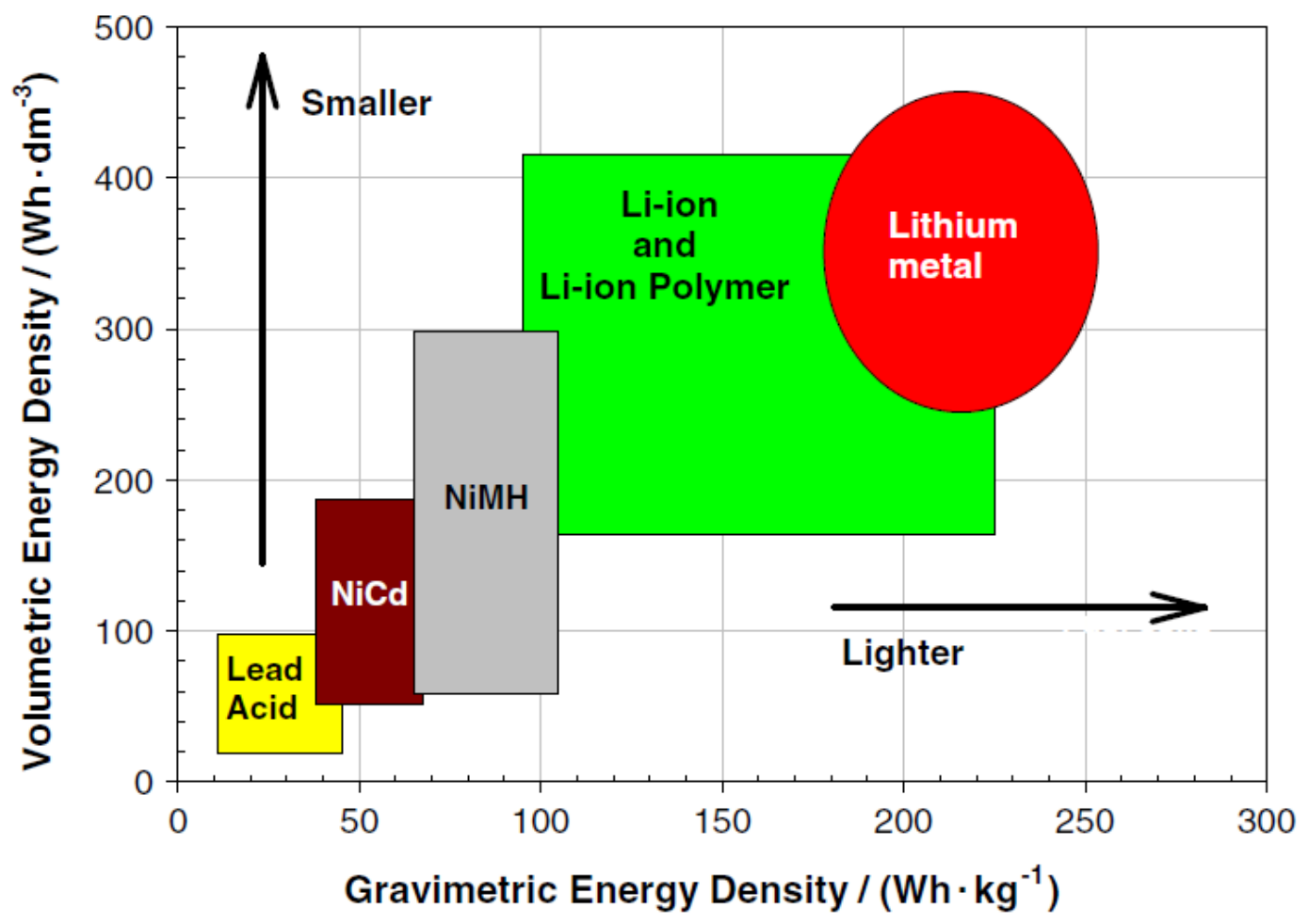

Figure 1. Volumetric energy density vs gravimetric energy density of commonly used rechargable batteries.

Although lithium based rechargeable batteries possess ideally performance theoretically, the commercialization of lithium based rechargeable batteries is still in the initial step. There are three promising candidates probably be industry standards in the future, which concludes lithium-metal battery, lithium-ion battery with liquid electrolyte and lithium-ion battery with solid-state 
electrolyte. Lithium-metal battery, among all the three kinds of lithium-based batteries, can offer the highest energy density. Theoretically, the gravimetric energy density of lithium-metal battery can reach $150 \mathrm{Wh} / \mathrm{kg}$, and the volumetric energy density can reach $300 \mathrm{Wh} / \mathrm{liter}$. Two major problems resistive the development of lithium-metal batteries. First, safety problem. Metallic lithium is a highly reactive element, this reason leads to rechargeable lithium-metal batteries too risky in the practically application. Second, dendrites will happen at the surface of the separator between positive and negative electrodes after repeated charge and discharge. The result of dendrites is shortcut of the whole circuit. Lithium ion batteries with liquid electrolyte have been commercialized more than one decade because of the high performance, however, there are still some disadvantages of this kind of batteries. The most important one is the grave problem. Lithium electrolyte have to be packed in thick metal to protect the liquid leakage, so this kind of batteries are relative heavier and thicker compared with the one which use solid state electrolyte. Due to all the drawbacks of the lithium metal batteries and lithium ion batteries we need to improve, research start to focus on all solid state lithium ion batteries. Several important advantages of all solid state lithium ion battery attract people's attention. The first one is safety. For the lithium batteries with solid state electrolyte, leakage and explosive are not the problems. Second is the volume minimize. The electrolyte part of the all solid state lithium ion battery has the highest energy density compare to the liquid electrolyte. Because of the energy efficiency, solid state lithium ion batteries always packed in a series to enhance the voltage and capacity. Final one is the environmental friendly. Lead acid batteries and nickel batteries seriously damaged the environment at the beginning of those batteries put in practically application. The carbonate anode, metal oxide cathode and the ceramic electrolyte of the all solid state lithium ion battery all benign for the environment. To sum up, all solid state lithium ion battery is a promising candidate to commercialize in the future. 
The performance of all solid state lithium-ion batteries depends on all parts of materials which the batteries used. For the electrode part, development should focus on higher energy density, faster discharge kinetics and more stable. For the electrolyte part, inorganic solid state electrolytes have the trend replace liquid organic electrolytes since the safety, durability problem, negligible electronic conductivity and stable chemical reaction with anode and cathode materials. Furthermore, solid state electrolytes have free dendrite with lithium anode, which is utilized to lithium rechargeable batteries. It's worth noting that lithium rechargeable batteries received lots of attention since their high specific capacities. Previously, liquid electrolytes were commonly used in commercial lithium ion batteries, since the conductivities are higher than most inorganic electrolytes. However, unsafety problems such as explosion, fire accidents, leakage and thermal unstable driving force in the development of solid state inorganic electrolyte. The inverse characteristics are the advantages of a solid state electrolyte. Another reason solid state electrolytes have to take into account is the miniature of volume for commercial lithium batteries, which is good for vary the batteries form and design [2-8].

1.2 Oxide compounds as solid state electrolytes

For all solid state lithium ion batteries, the most important part is the electrolyte. Inorganic ceramics and organic polymers are two major classes of materials used for lithium ion batteries electrolyte. Aside ionic conductivity of these two kinds of materials, the main difference between the ceramics and the polymers is the mechanical properties. Inorganic ceramics have high moduli, so they are suitable for fabricate rigid battery, though more expensive for fabricating. On the other hand, ceramics are applicable at elevated temperature. However, low ionic conductivity and interfacial instability are the two foremost disadvantage of solid state electrolytes. 
Among the inorganic ceramic electrolytes, sulfide, phosphate and oxide compounds are widely investigated. Sulfide glasses or glass-ceramics hold the highest ionic conductivity, mostly higher than oxide glasses. But as common sense, sulfide is really easy to absorb moisture. So all the sulfide based glasses have to prepared in argon fill glove box [9-16]. Ionic conductivity of phosphate based glasses or glasses-ceramic usually one order lower than oxide glasses [17-23]. For all the lithium-ion conducting oxide systems, $(\mathrm{La}, \mathrm{Li}) \mathrm{TiO}_{3}$ is the most promising one. Table 1 list the properties of commonly used inorganic solid lithium ion conductor electrolytes. From table 1, we can see among all the lithium ion conductors, $\mathrm{La}_{2 / 3-\mathrm{x}} \square_{1 / 3-2 x} \mathrm{Li}_{3 \mathrm{x}} \mathrm{TiO}_{3}$ (LLTO) has the highest room temperature ionic conductivity and lowest activation energy. So we chose to investigate LLTO as the electrolyte for all solid state lithium ion battery.

Table 1. Summary of inorganic solid lithium ion conductor.

\begin{tabular}{|c|c|c|c|c|}
\hline $\begin{array}{l}\text { Electrolyte } \\
\text { name }\end{array}$ & Type & Typical composition & $\begin{array}{l}\text { RT ionic } \\
\text { conductivity } \\
(\mathrm{S} / \mathrm{cm})\end{array}$ & $\begin{array}{l}\text { Activation } \\
\text { energy (eV) }\end{array}$ \\
\hline LLTO & Crystalline & $\mathrm{La}_{2 / 3-x} \square 1 / 3-2 x \mathrm{Li}_{3 x} \mathrm{TiO}_{3}$ & $10^{-3}$ & $0.3-0.4$ \\
\hline NASICON & Crystalline & $\mathrm{Li}_{1.3} \mathrm{Al}_{0.3} \mathrm{Ti}_{1.7}\left(\mathrm{PO}_{4}\right)_{3}$ & $3 \times 10^{-3}$ & $0.3-0.5$ \\
\hline LISICON & Crystalline & $\mathrm{Li}_{14} \mathrm{ZnGe}_{4} \mathrm{O}_{16}$ & $10^{-6}$ & $0.4-0.6$ \\
\hline Thio-LISION & Crystalline & $\mathrm{Li}_{3.4} \mathrm{Si}_{0.4} \mathrm{P}_{0.6} \mathrm{~S}_{4}$ & $6.4 \times 10^{-4}$ & $0.5-0.6$ \\
\hline Garnet & Crystalline & $\mathrm{Li}_{6} \mathrm{La}_{2} \mathrm{BaTa}_{2} \mathrm{O}_{12}$ & $4 \times 10^{-5}$ & $0.4-0.6$ \\
\hline Sulfide glass & Amorphous & $\mathrm{GeS}_{2}+\mathrm{Li}_{2} \mathrm{~S}+\mathrm{LiI}+\mathrm{Ga}_{2} \mathrm{~S}_{3}$ & $10^{-3}$ & $0.4-0.5$ \\
\hline LiPON & Amorphous & $\mathrm{Li}_{2.88} \mathrm{PO}_{3.73} \mathrm{~N}_{0.14}$ & $3.3 \times 10^{-6}$ & $0.5-0.6$ \\
\hline
\end{tabular}




\subsection{Properties and structures of LLTO}

$\mathrm{La}_{2 / 3-x} \square_{1 / 3-2 x} \mathrm{Li}_{3 x} \mathrm{TiO}_{3}$ (LLTO) was first discovered by Yoshiyuki Inaguma’s group in 1992 [24]. From the ICP data, the molar ratios of LLTO metals La:Li:Ti=0.34:0.51:1, content sum of La and Li less than 1, so the A-site vacancies were introduced. A.C. impedance method was used to measure the ionic conductivity and D.C. method was used to test the electronic conductivity. The total ionic conductivity of LLTO was higher than $2 \times 10^{-5} \mathrm{~S} \mathrm{~cm}^{-1}$ at room temperature, which is the summation of the ionic conductivity in the grain bulk, $1 \times 10^{-3} \mathrm{~S} \mathrm{~cm}^{-1}$, and the ionic conductivity at grain boundary, $7.5 \times 10^{-5} \mathrm{~S} \mathrm{~cm}^{-1}$. The calculated activation energies below $400 \mathrm{~K}$ were $0.15 \mathrm{eV}$ and $0.4 \mathrm{eV}$ respectively.

The structure of LLTO is cubic perovskite with cell parameter 3.8710(2) A. Figure 2 shows a $2 \times 2 \times 2$ supercell of LLTO. From figure 2 , we can see A-site vacancies exist in this structure. Lithium ions are allowed to migrate freely in these vacancies. This migration mechanism leads the high ionic conductivity of LLTO.

Structures change under different sinter conditions:

Before we talk about the different structures of LLTO under different sinter conditions, we need to explain $\mathrm{TiO}_{6}$ octahedra tilt in different LLTO structures. Figure 3 is the LLTO structure we built from our experimental data and geometry optimized by Materials Studio 6.0. After geometry optimize, $\mathrm{TiO}_{6}$ octahedra tilt takes place. Goldschmidt tolerance factor $(\mathrm{t})$ can be used to describe the octahedra tilt degree in perovskite structure as shown in equation (1): 


$$
t=\frac{r_{A}+r_{0}}{\sqrt{2}\left(r_{B}+r_{0}\right)}
$$

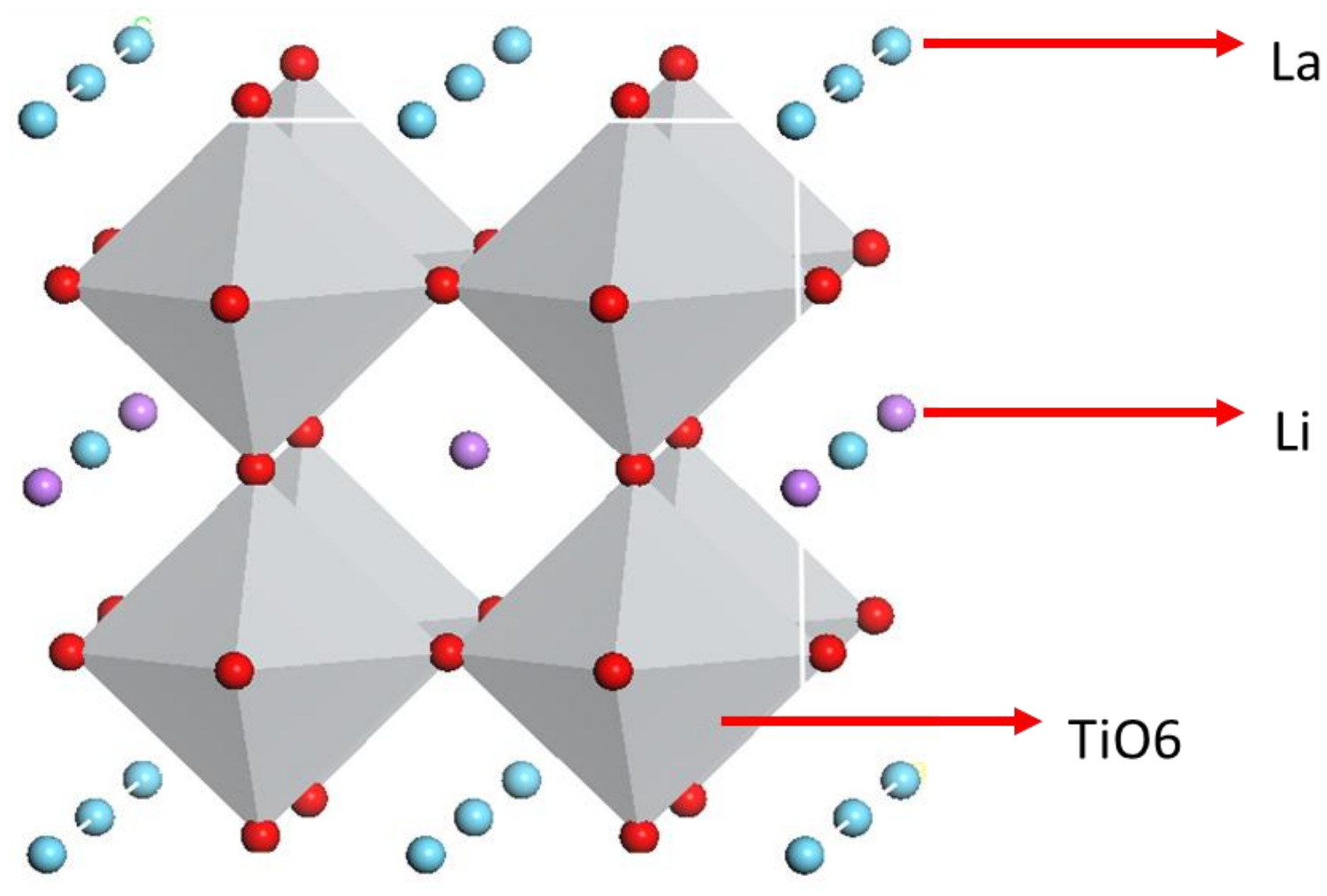

Figure 2. Schematic diagram of LLTO $2 \times 2 \times 2$ single perovskite supercell (Purple balls, blue balls and red balls are lanthanum, lithium and oxygen, titanium is in the grey $\mathrm{TiO}_{6}$ octahedra).

In the above equation, $r_{A}$ is the radius of the A-cation, $r_{B}$ is the radius of $\mathrm{B}$-cation and $r_{0}$ is the radius of the anion. For cubic structures, $t$ usually arrange from 0.9 to one, and the octahedra tilt in our cubic perovskite LLTO is slightly. For the orthorhombic and rhombohedral structures, $0.71<\mathrm{t}<0.9$. If the A site ions are too small, the tilt of octahedral will be more seriously. 
As previously mentioned, Inaguma et al. used experimental data compared with simulated powder X-ray diffraction data to determine his LLTO structure. His result shows that lanthanum ions and lithium ions are ranging in a double perovskite structure along the $c$ direction $\left(c=2 a_{p}\right)$, where $a_{p}$ corresponds the cubic perovskite cell parameter. That means along the $c$ direction,

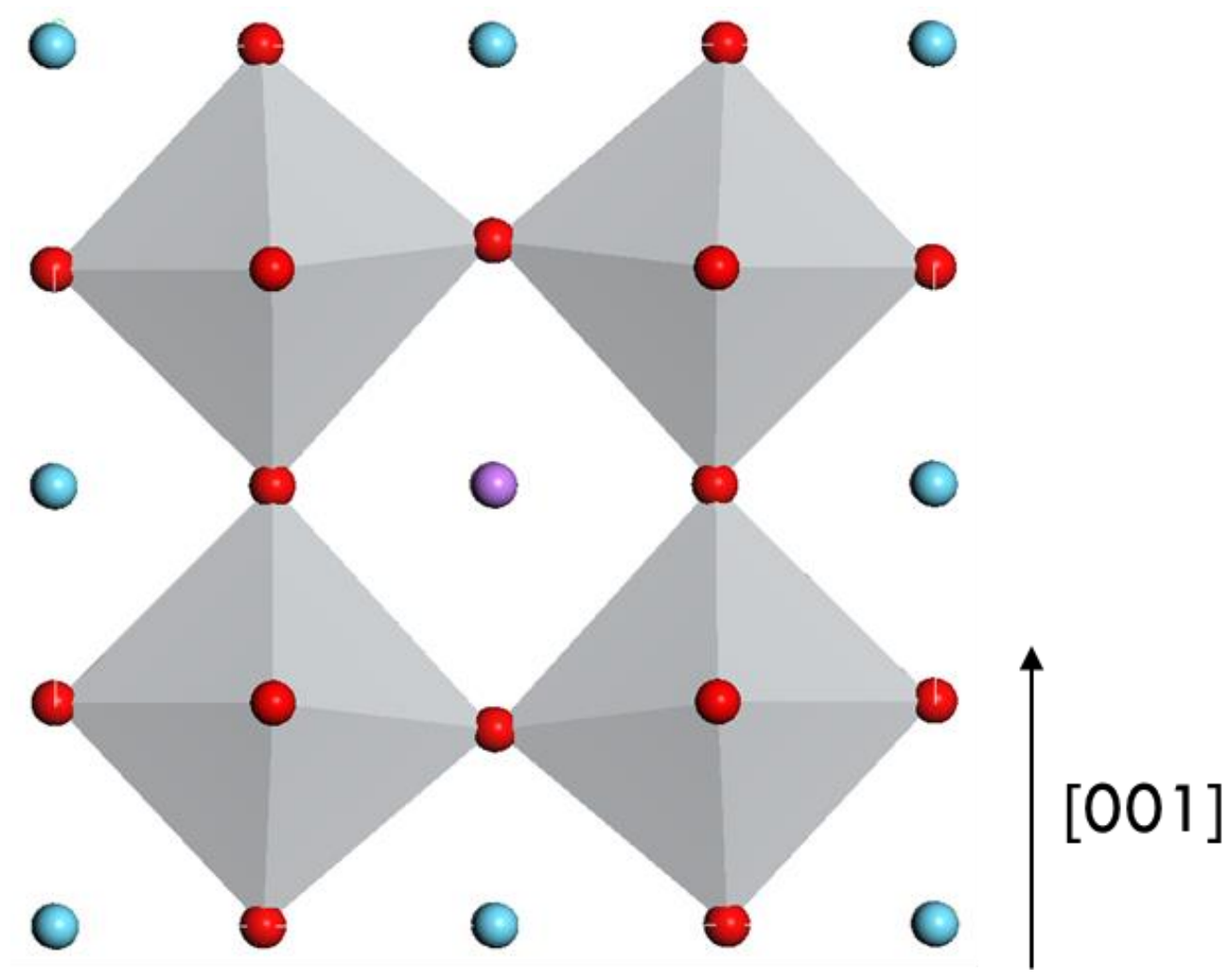

Figure 3. Perovskite structure of LLTO in [100] direction (Purple balls, blue balls and red balls are lanthanum, lithium and oxygen, titanium is in the grey $\mathrm{TiO}_{6}$ octahedra).

lanthanum ion rich layer and lithium ions layer are alternately [24]. A. Varez first proposed the concept "diagonal perovskite". Synthesis method was calcine LLTO powder at $1150^{\circ} \mathrm{C}$ for 12 hours then pressed into pellets and fired at $1350^{\circ} \mathrm{C}$ for 6 hours. He used TEM to observe LLTO in [110] direction and defined [110] direction as the unit cell axis direction which shows in figure 4. 
In the cubic perovskite, $a=a_{p}=3.8 \AA$. So for the diagonal perovskite, $a=2^{1 / 2} a_{p}=5.4 \AA, c=2 a_{p}$ [25]. J.L. Fourquet et al. investigated LLTO composition range $0.06<x<0.14$. He heated LLTO three times for 10 hours at $1150{ }^{\circ} \mathrm{C}$ and cool down in furnace. Structure of LLTO they synthesized was tetragonal structure (P4/mmm, $\left.a=a_{p}, c \sim 2 a_{p}\right)$, as $x$ increase, $c / 2 a$ decrease since the radius of the lithium ion is much smaller than lanthanum ion, shown in figure 5 [26]. Powder neutron-diffraction and Rietveld method refinement are good ways to test atoms positions in a structure. Due to the tilt of $\mathrm{TiO}_{6}$ octahedra in LLTO causes a doubling of the basic unit-cell, Inaguma et al. used furnace-cooled sinter method to synthesized lithium-deficient LLTO and claimed the orthorhombic structure [27]. Figure 6 shows the orthorhombic perovskite structure of LLTO from powder neutron-diffraction data. From figure 6, we can see lithium ions are located off A-site and close to the lanthanum ions. The octahedral tilt strongly affect the lithium ions pathways in LLTO and we expect lithium ions migrate in only $a$ and $b$ directions which we will talk about later. One year later, A. Varez and Y. Inaguma collaborated to investigate lithium rich, $3 x=0.5$, quenched LLTO structure [28]. LLTO was quenched at $1600 \mathrm{~K}$ into liquid $\mathrm{N}_{2}$. They also used neutron diffraction to study the quenched LLTO structure. Rhombohedral structure (space group: $R \overline{3} c$ ) which $a=b=\sqrt{2} a_{p}, c=2 \sqrt{3} a_{p}$ was found. Figure 7 shows rhombohedral structure of LLTO. From figure 7, we can see lanthanum vacancies are disordered, so lithium ions can migrate in 3 directions in this structure because no obvious lanthanum deficient layers and lanthanum rich layers which lithium ions are difficult to move through exist in this structure. Same year, Jesus Sanz et al. investigated orthorhombic perovskite LLTO structure change from 5K to 773K [29]. At low temperature, lanthanum rich and deficient layers alternate along [001] direction and $\mathrm{TiO}_{6}$ 

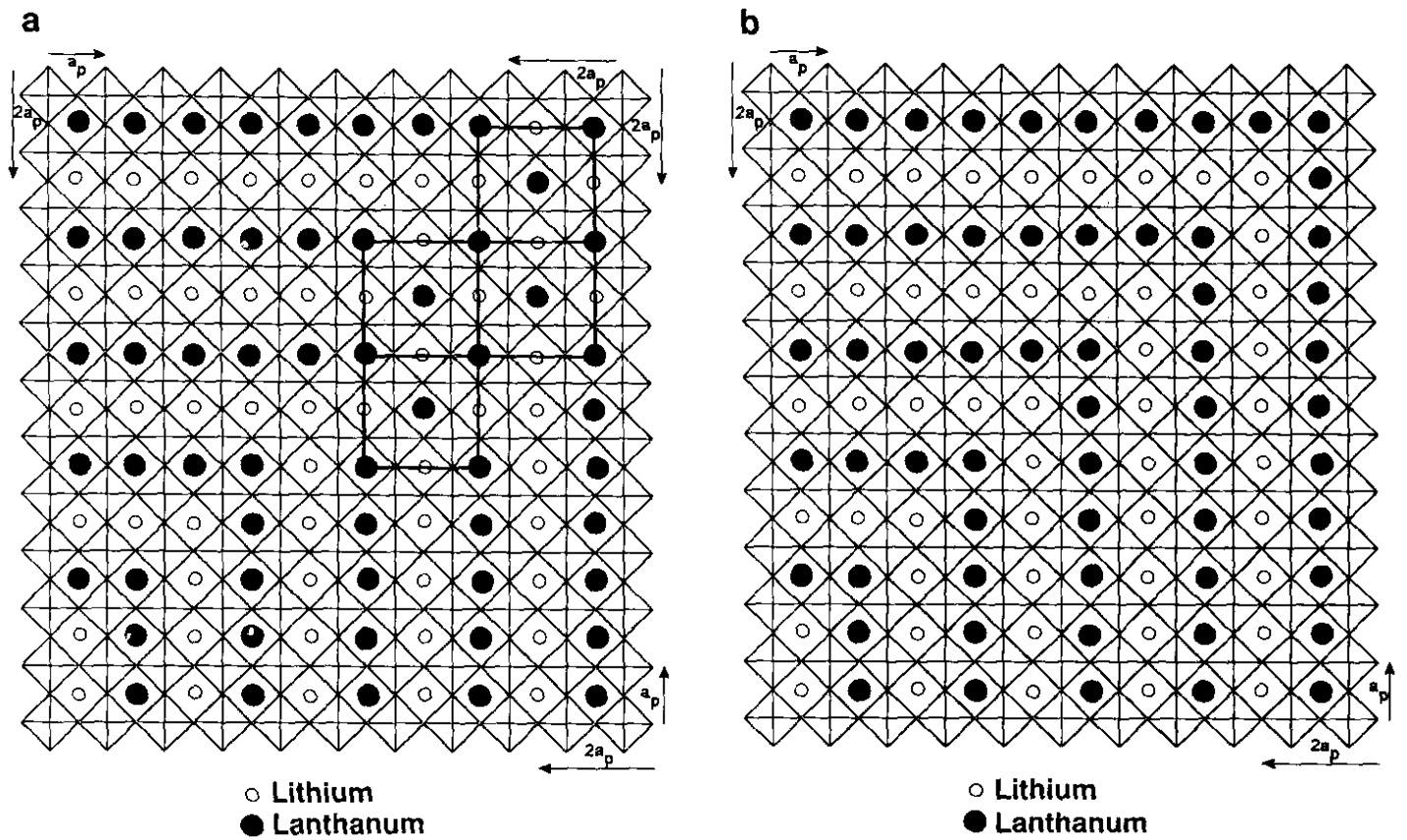

Figure 4. [110] domains of a LLTO diagonal perovskite cell.

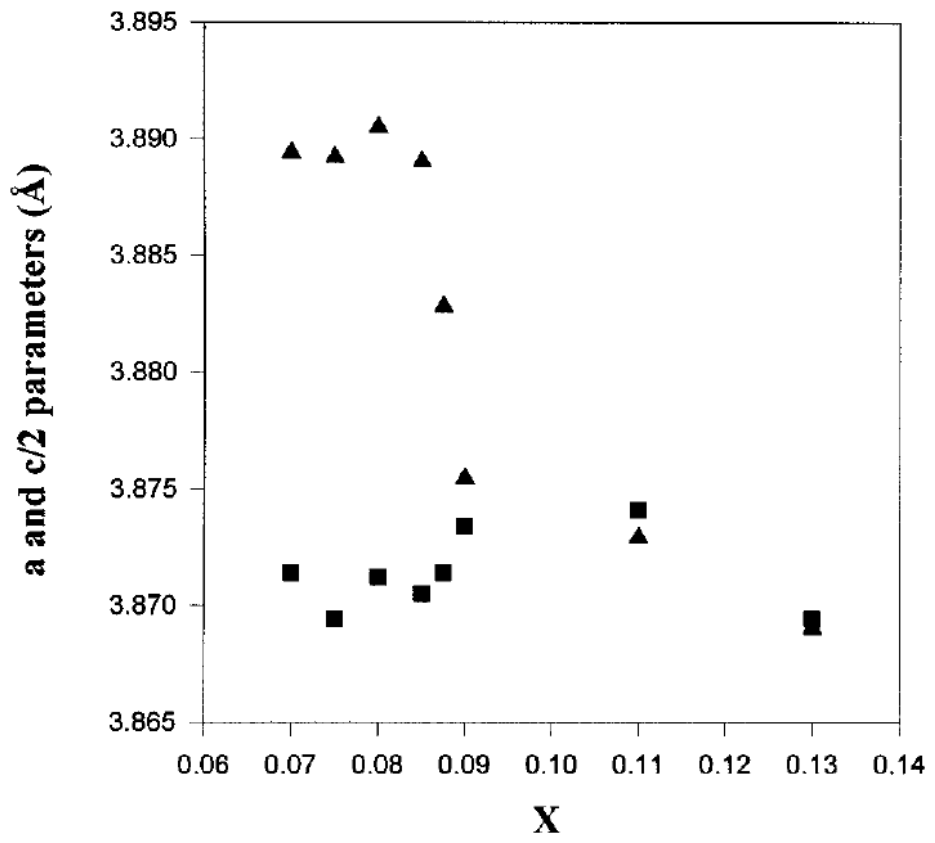

Figure 5. Evolution of the unit cell parameters versus $x$ (a, squares; $c / 2$, triganles). 


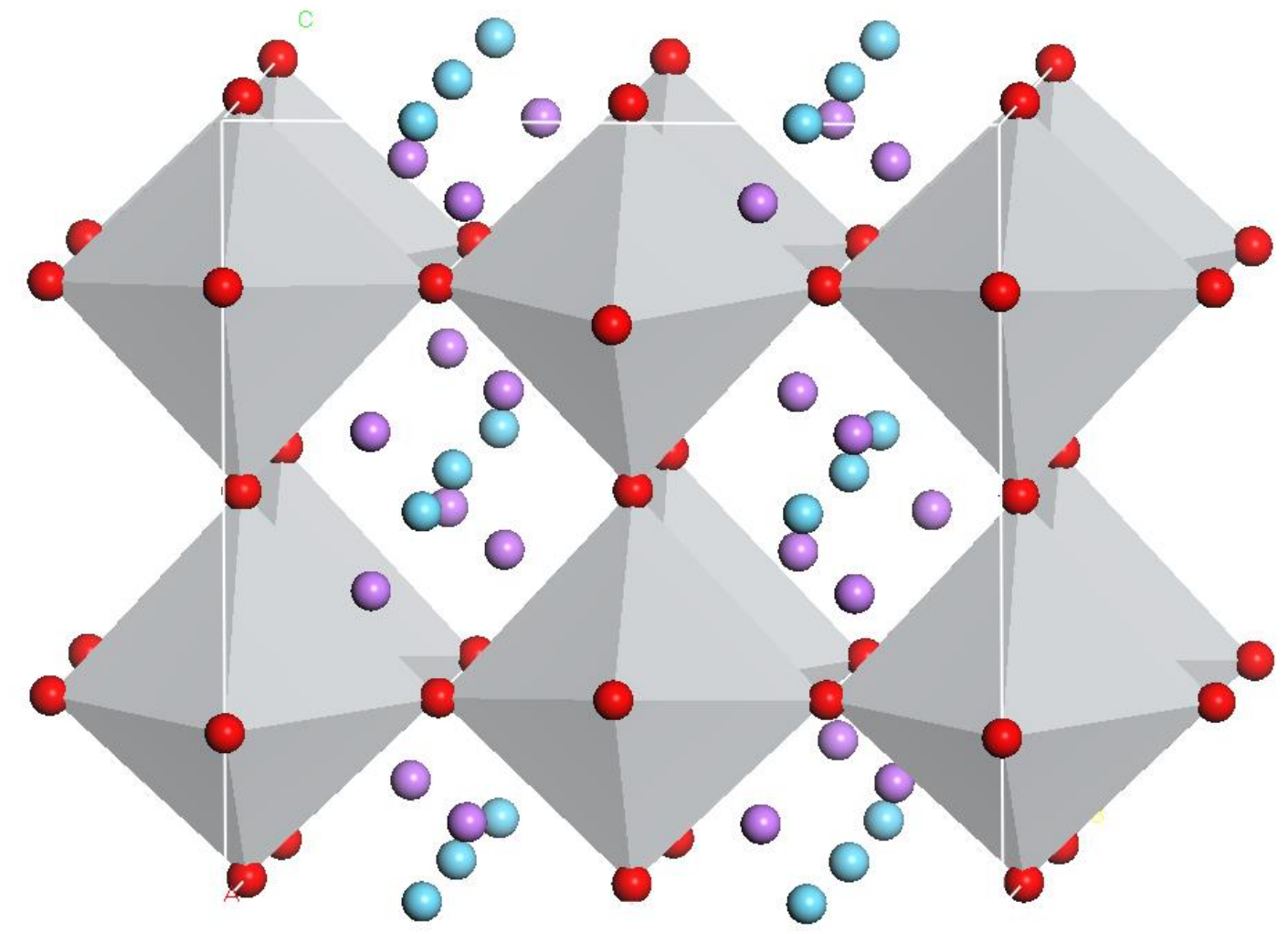

Figure 6. A structure from the perspective of the $\mathrm{TiO}_{6}$ octahedral framework (Purple balls, blue balls and red balls are lanthanum, lithium and oxygen, titanium is in the grey $\mathrm{TiO}_{6}$ octahedra).

octahedral tilted around $b$-axis. As the temperature increase, $\mathrm{TiO}_{6}$ octahedral tilt becomes less obvious and the structure of LLTO turns to tetragonal phase (Space group $P 4 / \mathrm{mmm}$ ). Structure disorder is always happened on quenched LLTO since at high temperature, the structure is not stable and the cooling rate is so high. Due to the structure disorder and tilt of $\mathrm{TiO}_{6}$ octahedral, for the quenched LLTO, lithium ions prefer to migrate in three directions. Annealing or furnace cooled LLTO always has stable double perovskite structure. Since lanthanum rich layers and vacancies rich layers are alternated, theoretically lithium ions pathways are two directions, along $a$ and $b$ directions.

Valence change of titanium: 
LLTO was evolved from its original material $\mathrm{LaTiO}_{3}$. $\mathrm{LaTiO}_{3}$ belongs to $\mathrm{RMO}_{3}$ single cubic perovskite structure ( $\mathrm{R}$ is a rare-earth ion, $\mathrm{M}=$ transition metal). The valence of lanthanum ion is

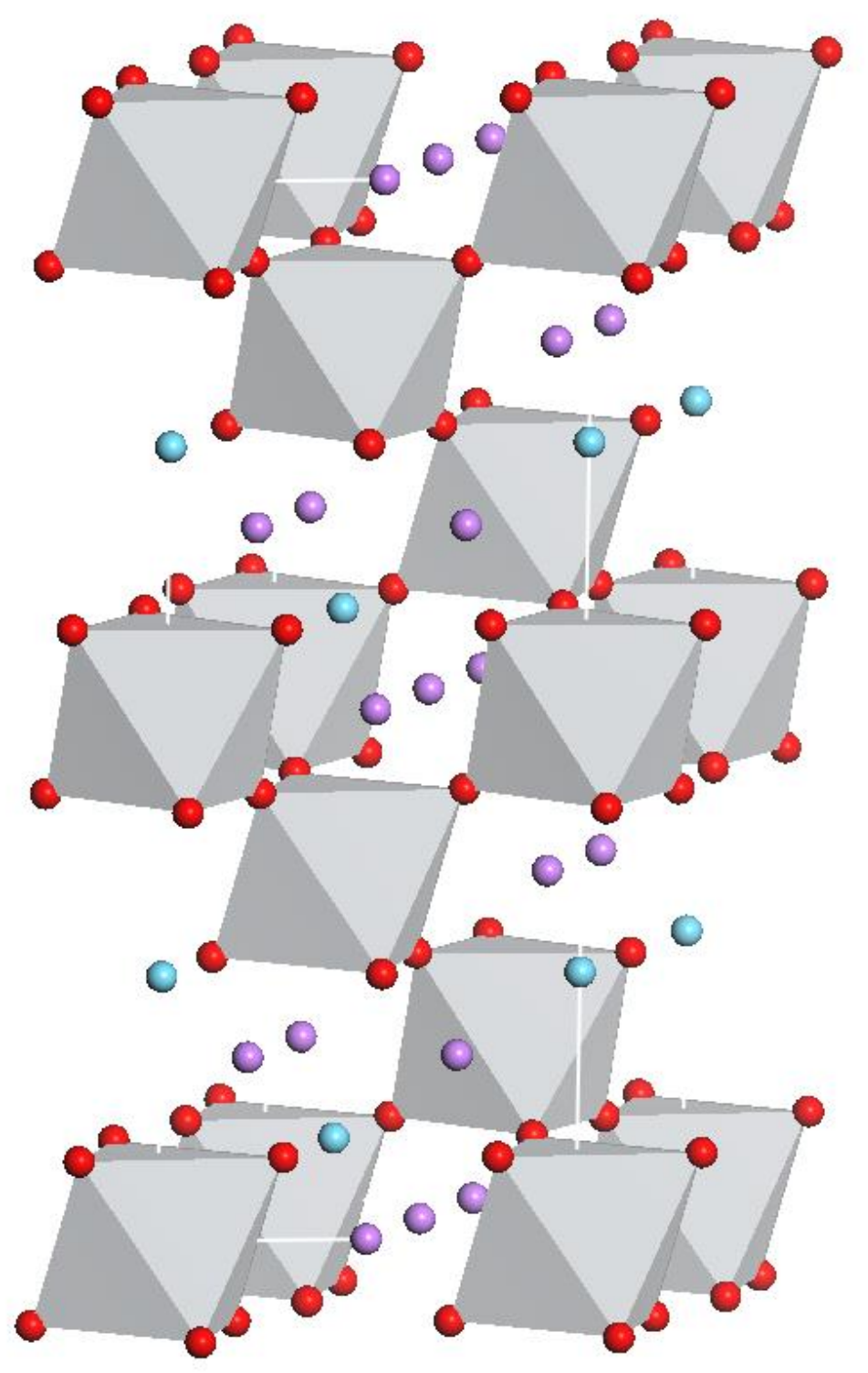

Figure 7. Rhombohedral structure of quenched LLTO (Purple balls, blue balls and red balls are lanthanum, lithium and oxygen, titanium is in the grey $\mathrm{TiO}_{6}$ octahedra).

$3+$. However one third lanthanum vacancies exist in $\mathrm{LaTiO}_{3}$, and the valence of titanium is $4+$, but the $3 d$ orbital electron ion of $\mathrm{Ti}^{4+}$ is very weak $[30,31] . \mathrm{La}_{2 / 3-x} \square_{1 / 3-2 x} \mathrm{Li}_{3 x} \mathrm{TiO}_{3}$ is lithium inserting 
into lanthanum vacancies, oxidation of $\mathrm{Ti}^{4+}$ converted to $\mathrm{Ti}^{3+}$ maybe take place during in this procedure. Figure 8 illustrate the process of small quantity of lithium insert in to $\mathrm{LaTiO}_{3}$. From figure 8, we can see when small quantity of lithium insert in to $\mathrm{LaTiO}_{3}$, lithium located into the lanthanum A-site vacancies, which equation (2) is interpretable. When certain amount of lithium inserted in to $\mathrm{LaTiO}_{3}$ and lithium insertion continue, electrochemical reaction will happen and the valence of titanium start to reduce.

$$
\frac{x}{2} \mathrm{Li}_{2} \mathrm{CO}_{3}+\frac{2-x}{6} \mathrm{La}_{2} \mathrm{O}_{3}+\mathrm{TiO}_{2} \rightarrow \mathrm{Li}_{x} \mathrm{La} \frac{2-x}{3} \mathrm{TiO}_{3}+\frac{x}{2} \mathrm{CO}_{2}
$$
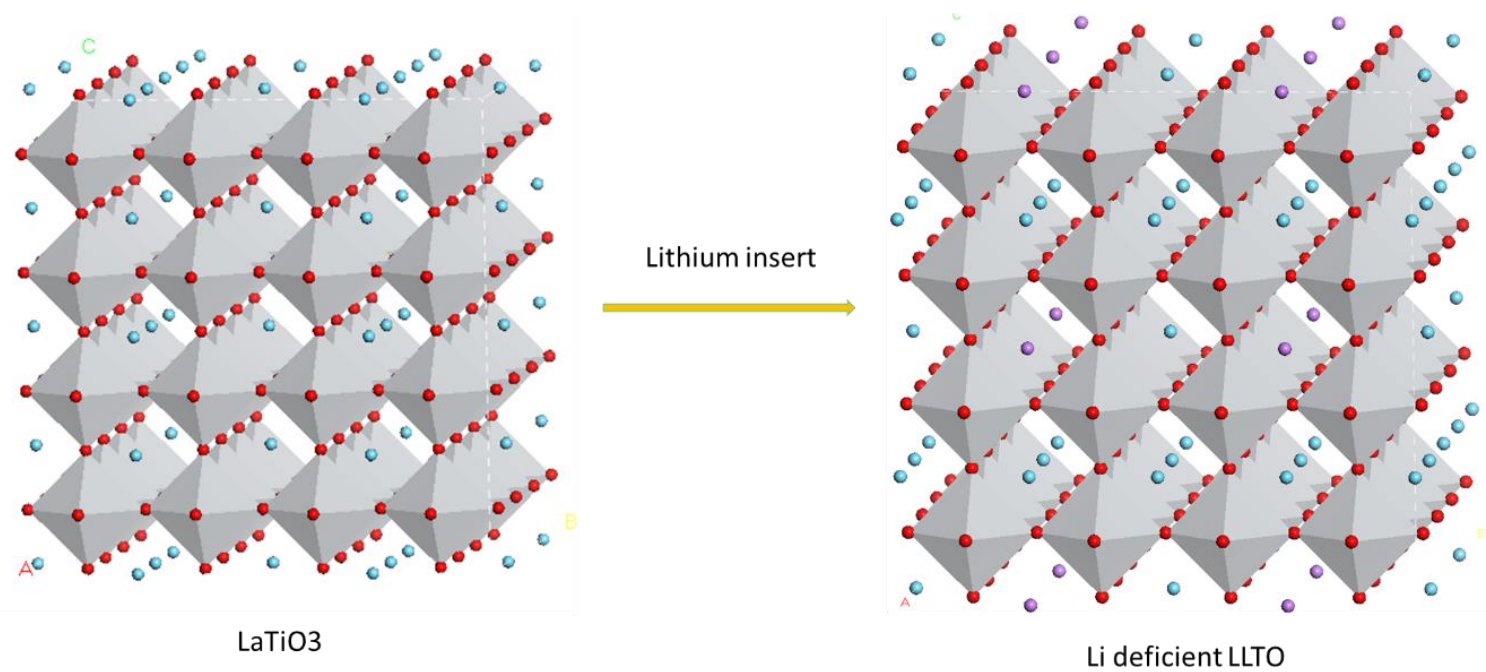

Figure 8. Process of small quantity of lithium insert in to $\mathrm{LaTiO}_{3}$ (Purple balls, blue balls and red balls are lanthanum, lithium and oxygen, titanium is in the grey $\mathrm{TiO}_{6}$ octahedra).

This time, lithium will no longer dope into the A-site vacancies, but in the interstitial. Equation (3) illustrate the process of large amount of lithium insert in to LLTO. From equation (3), when $y>0$, the valence of titanium will be lower than 4 . Figure 9 is the lithium rich LLTO structure from neutron diffraction data [32]. 


$$
\mathrm{yLi}^{+}+\mathrm{ye}^{-}+\mathrm{Li}_{\mathrm{x}} \mathrm{La}_{3} \frac{2-x}{3} \mathrm{TiO}_{3} \rightarrow \mathrm{Li}_{\mathrm{x}+\mathrm{y}} \mathrm{La}^{2-x} \frac{2-x}{3} \mathrm{TiO}_{3}
$$

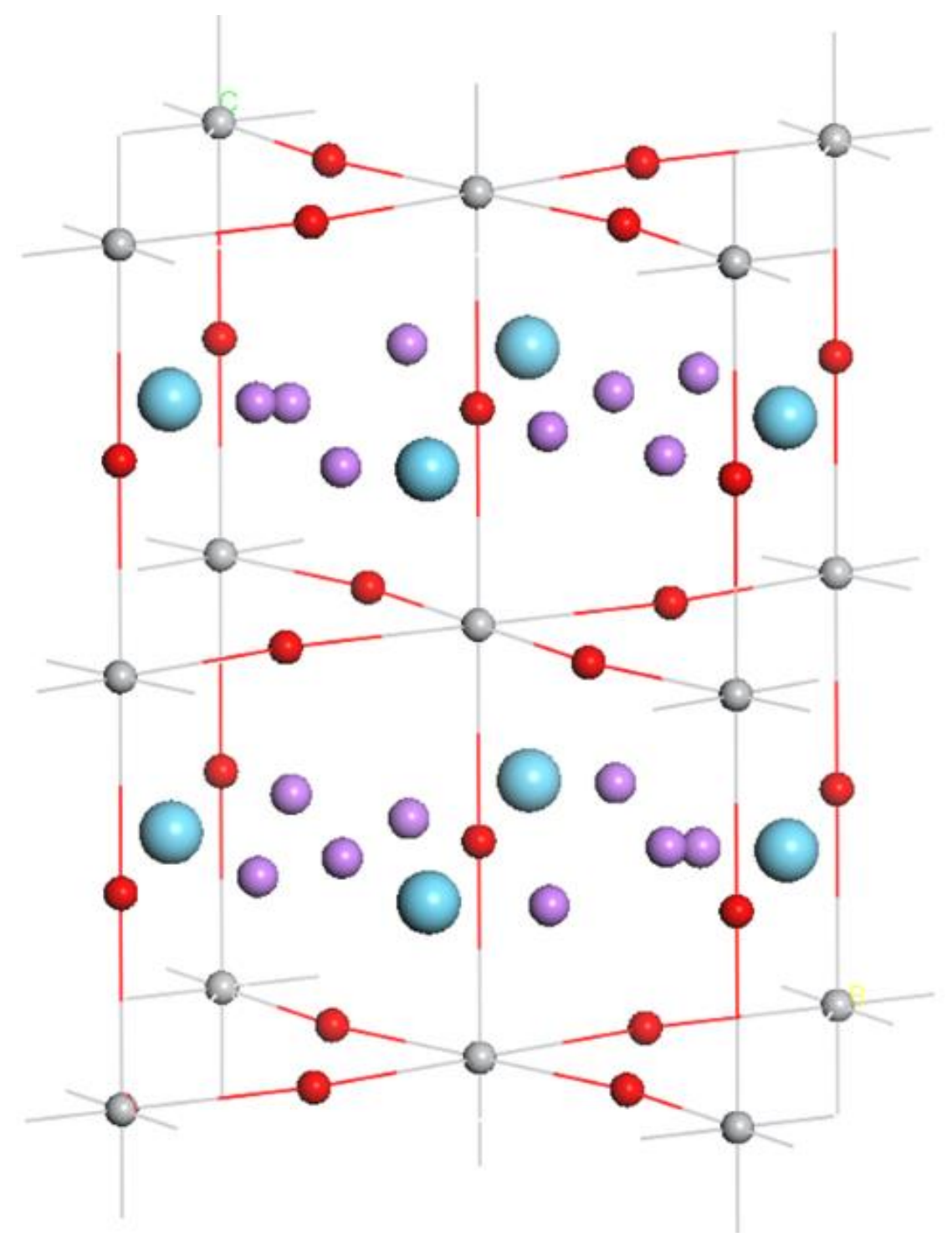

Figure 9. Lithium rich structure of LLTO (Purple balls, blue balls and red balls are lanthanum, lithium and oxygen, titanium is in the grey $\mathrm{TiO}_{6}$ octahedra). 
Titanium reduction is the solely concern that LLTO is not suitable for used as electrolyte since oxidation of $\mathrm{Ti}^{4+}$ increase the electronic conductivity when react with metallic lithium. KaiYun Yang et al. used X-ray photoelectron spectroscopic and secondary ion mass spectroscopic to test the titanium valence change of $11 \% \mathrm{La}^{3+} / \mathrm{Li}^{+}$-site vacant LLTO powder [33]. The result was $12 \% \mathrm{Ti}^{4+}$ converted to $\mathrm{Ti}^{3+}$ and this valence change can be controlled by changing the number of vacancies of LLTO. For avoid oxidation of $\mathrm{Ti}^{4+}$, we prefer to use lithium deficient LLTO. First, less $\mathrm{Ti}^{4+}$ valence change can be the case in lithium deficient LLTO. Second, as previously mentioned, the structure of lithium deficient LLTO is more stable. Finally, more lithium vacancies LLTO is good of lithium ions movement when LLTO used as electrolyte in lithium ion battery.

Ab-initio was commonly used to calculate the lithium ion pathways in LLTO. According to first principle calculation, lithium ions only migrate in $a$ and $b$ directions [34-41]. For generally speaking, people calculated the activation energies which lithium ions migrate in different directions, usually $a, b$ and $c$ directions. The direction of lowest activation energy of lithium ions is the pathway of the migration. Michele Catti used $\mathrm{Ab}$ initio simulation calculated lithium ions migration pathway and corresponding activation energy in lithium deficient LLTO [34]. The composition he assumed was $\mathrm{Li}_{0.125} \mathrm{La}_{0.625} \mathrm{TiO}_{3}$. Least common multiple for this composition is $\mathrm{Li}_{1} \mathrm{La}_{5} \square_{2}\left(\mathrm{TiO}_{3}\right)_{8} .2 a_{p} \times 2 a_{p} \times 2 a_{p}$ perovskite supercell was built and the number of lanthanum in each (001) layer combination was 4-1 and 2-3 as shown in figure 10. In model 1, lithium ions migrate in two dimensions with activation battier of $0.47 \mathrm{eV}$. Lithium ions migrate in one dimension in model 2 , the activation energy was $0.42 \mathrm{eV}$. For model 1 , in the $1 \mathrm{La}+1 \mathrm{Li}+1 \square$ layer, lithium ions take right angle turns when move in this layer, figure 11 illustrates the lithium ions migration in both model. Later, he used same method to calculate the activation energies of lithium ion movement in lithium rich LLTO structure [41]. Assumed composition was $\mathrm{Li}_{0.3} \mathrm{La}_{0.567} \mathrm{TiO}_{3}$ 


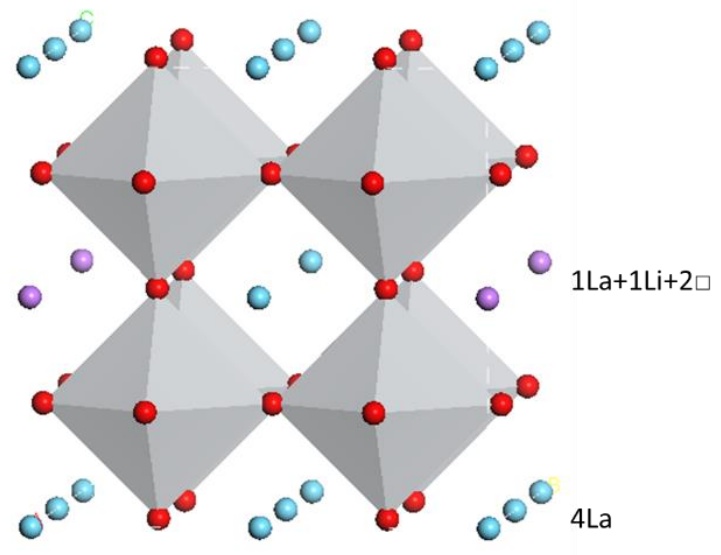

Model 1

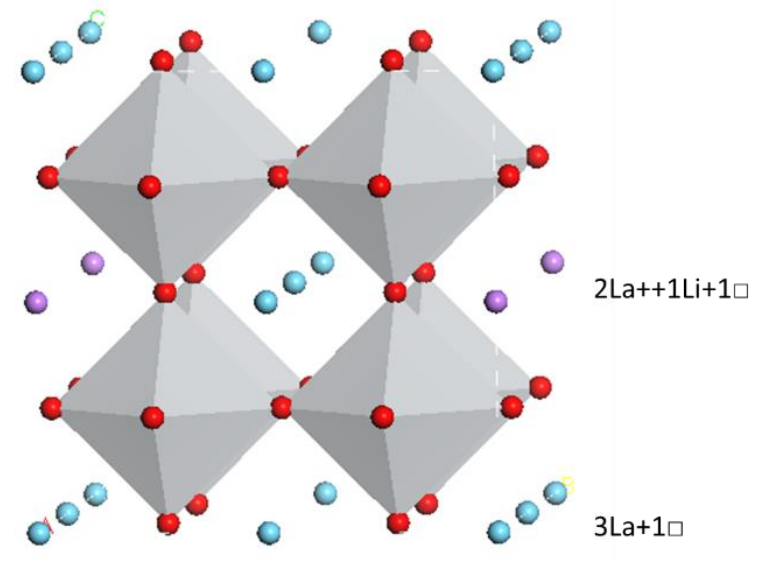

Model 2

Figure 10. Simulated lithium deficient perovskite structure supercell with different (001) layer combination (Purple balls, blue balls and red balls are lanthanum, lithium and oxygen, titanium is in the grey $\mathrm{TiO}_{6}$ octahedra).
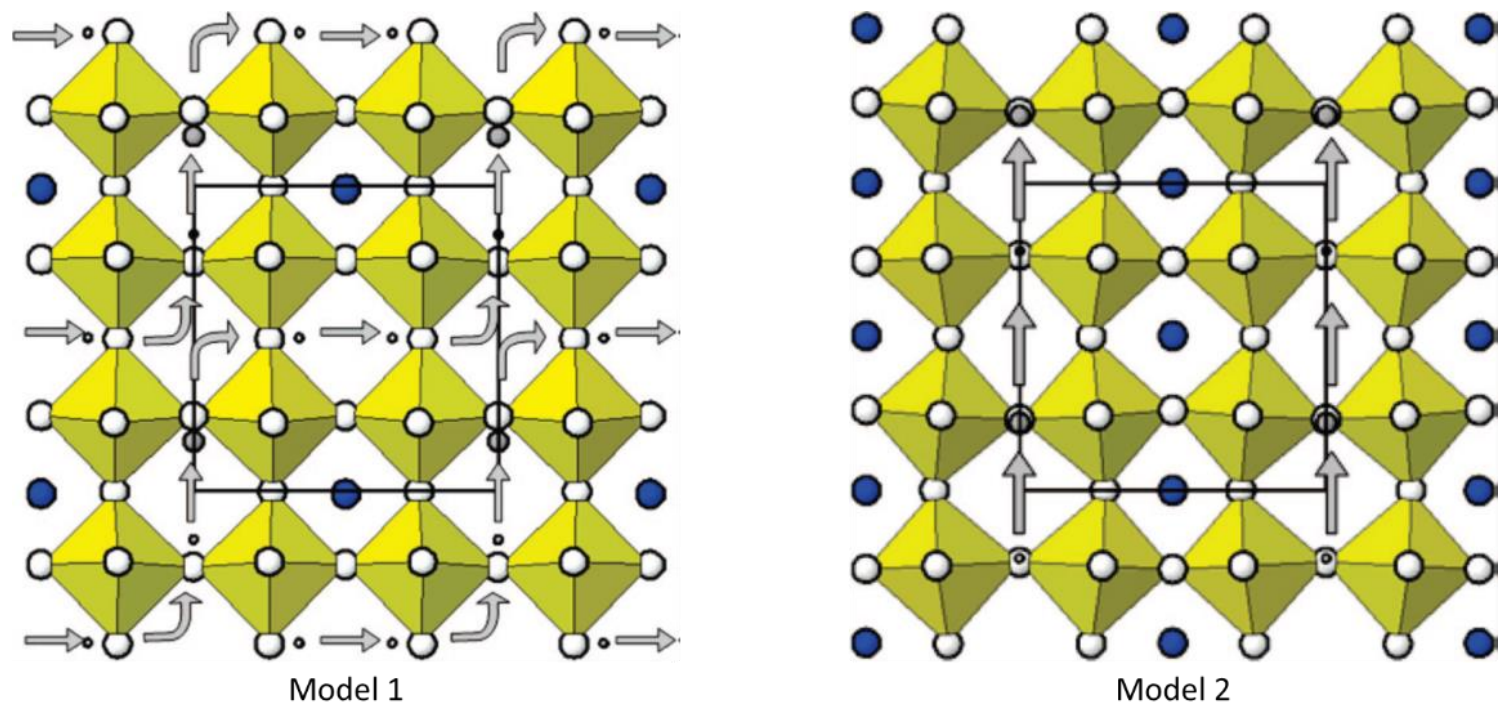

Figure 11. Lithium ion migration pathways in simulated lithium deficient LLTO.

$2 a_{p} \times 2 a_{p} \times 4 a_{p}$ perovskite supercell, the least common multiple of the elementary composition is $\mathrm{Li}_{5} \mathrm{La}_{9} \square_{2}\left(\mathrm{TiO}_{3}\right)_{16}$. Possible combinations of the number of lanthanum in each (001) layer conclude: 4-4-1-0,4-3-2-0,4-3-1-1,4-2-2-1, 3-3-3-0,3-3-2-1 and 3-2-2-2. Now we just talk about 3-3-2-1 
combination which showed in figure 12. In the $3 \mathrm{La}+1 \mathrm{Li}$ layers, lithium ions cannot move. In the 2La+2Li layers, lithium ions move in $1 \mathrm{D}$ pathways and the activation energy $0.31 \mathrm{eV}$. In the $1 \mathrm{La}+1 \mathrm{Li}+2 \square$ layers, lithium ions migrate in $2 \mathrm{D}$ pathways, however, the activation energy is significantly larger than in 1D pathways layers. The problem for this work is for the lithium rich LLTO structure, lithium ions should locate in the interstitial sites as previously we mentioned. In 2012, Dae-Hee Kim et al studied same lithium deficient LLTO composition as Michele Catti's simulation by density functional theory [39]. Different layers combinations were built and the activation barrier of lithium ions migrate in different structure was calculated as shown in figure 13. Structure a is the reference, so the activation energy is $0 \mathrm{eV}$. Structure be is the same structure of figure 10 model 1 , the activation energy coincide really good. Structure $\mathrm{f}$ is the same combination as figure 10 model 2 , the activation energy is higher than model 2 because lithium ions are surrounded by lanthanum atoms in [100] and [010] directions.

From all the simulations above, we can conclude that for reaching highest ionic conductivity and lowest activation energy of LLTO, we should try to control the atoms arrangement of (001) layers. Perfect atom arrangement of LLTO for conduct lithium ions is fully lanthanum atoms occupied layers and lanthanum deficient layers alternate, like the structure $a$ in figure 13. We will take effort on this orientation in the future.

Most people assumed lithium ion located off A-site and $\mathrm{TiO}_{6}$ octahedral tilt plays an important role in lithium ions movement. Valence of lithium ion is +1 and valence of $\mathrm{O} 4$ window is -8 , lithium ions trapped by $\mathrm{O} 4$ windows and $\mathrm{TiO}_{6}$ octahedra turned as lithium ion goes through the $\mathrm{O} 4$ window as shown in figure 14 [35] and figure 15 [39]. The $\mathrm{TiO}_{6}$ octahedra tilt and the $\mathrm{O} 4$ window trap lithium ions are the two main reasons lithium ions turn right angle when go through lanthanum deficient layers. 


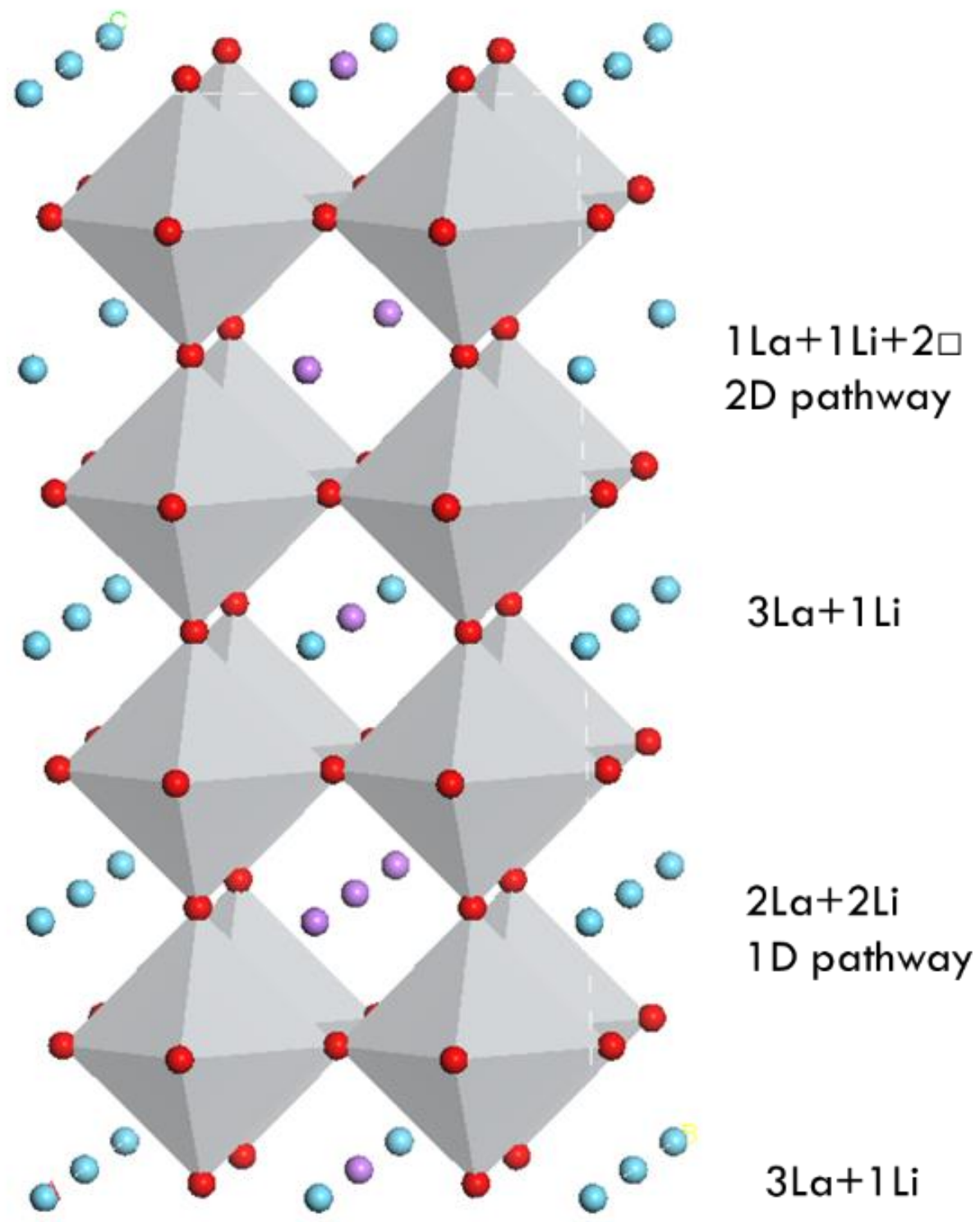

Figure 12. Lithium pathways for different (001) layer combinations (Purple balls, blue balls and red balls are lanthanum, lithium and oxygen, titanium is in the grey $\mathrm{TiO}_{6}$ octahedra). 

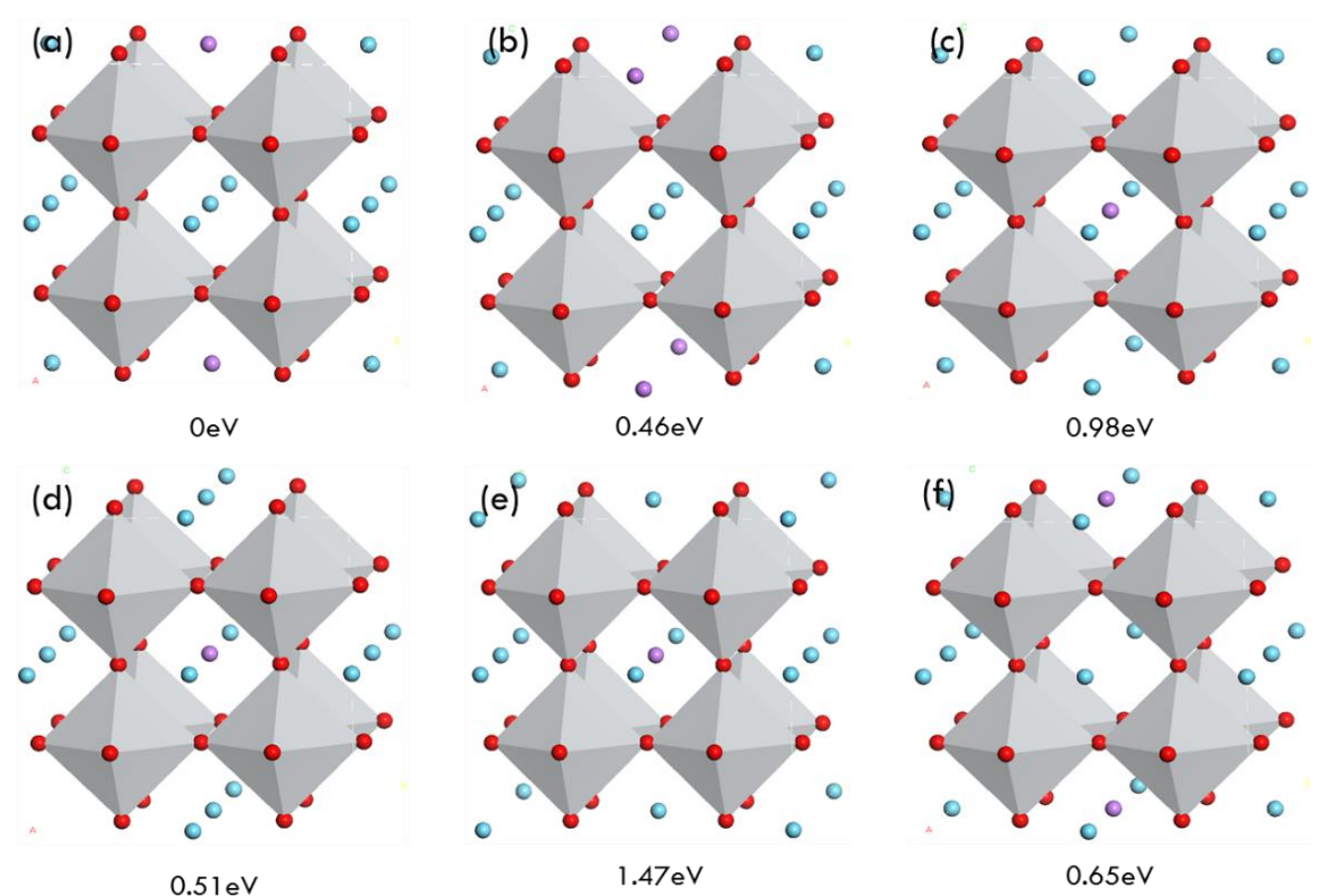

Figure 13. Calculated activation energies of various atom arrangements lithium deficient LLTO (Purple balls, blue balls and red balls are lanthanum, lithium and oxygen, titanium is in the grey $\mathrm{TiO}_{6}$ octahedra).

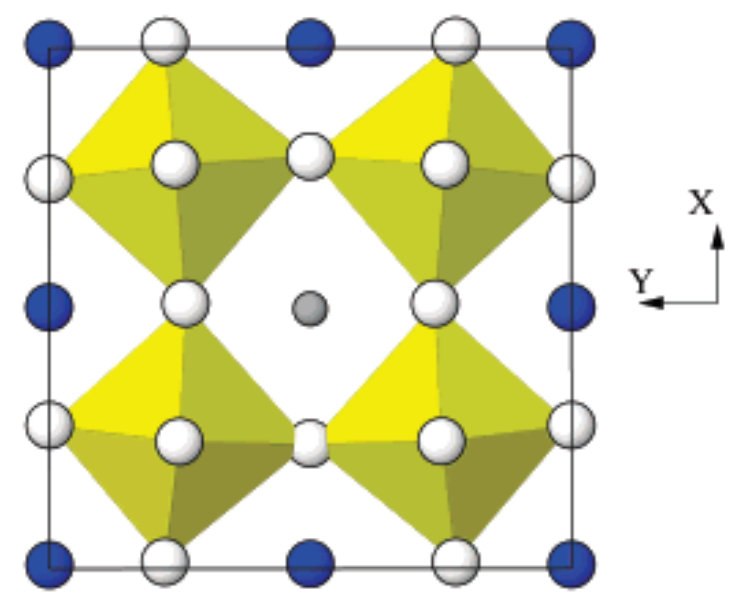

(a)

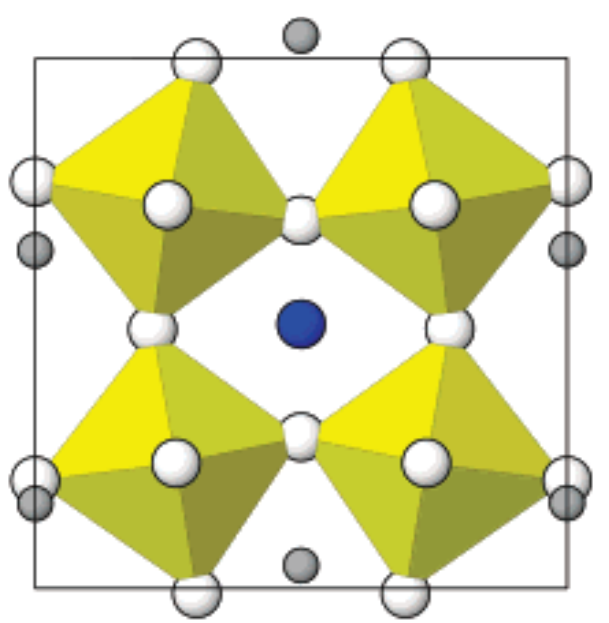

(b)

Figure 14. Schematic diagram of octahedral tilt during lithium ions go through $\mathrm{O} 4$ window (Grey balls, blue balls and white balls are lithium, lanthanum and oxygen, titanium is in yellow octahedral). 


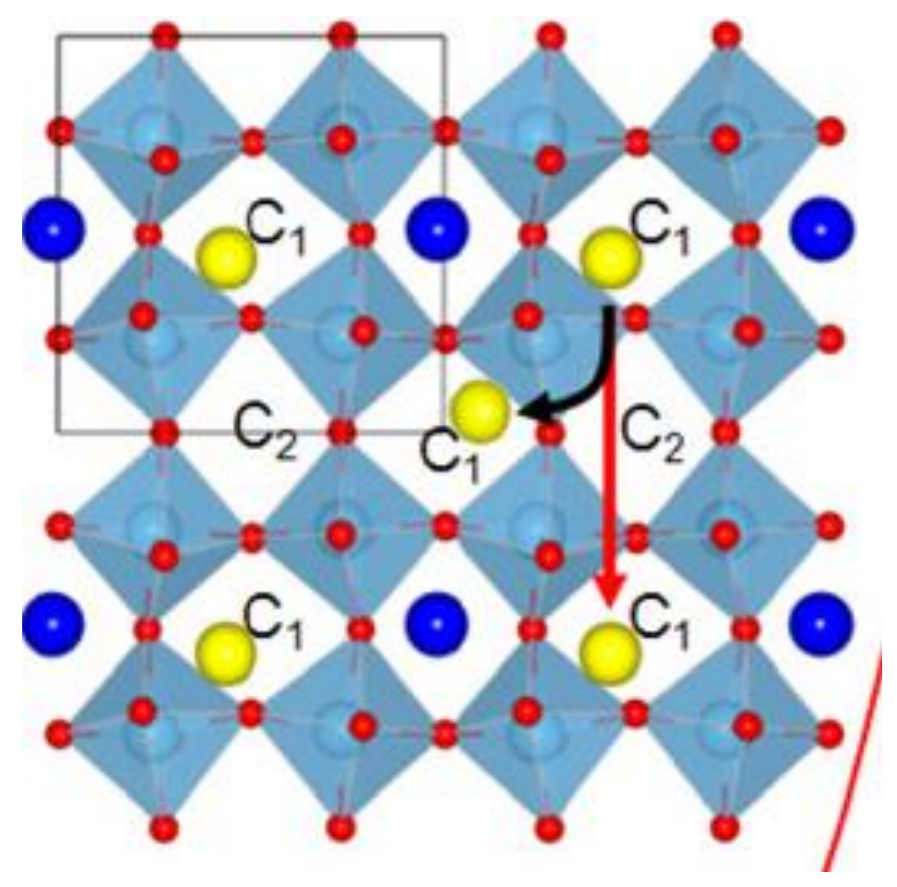

Figure 15. Octahedral tilt as lithium ions go through $\mathrm{O} 4$ window (Yellow balls, blue balls and red balls are lithium, lanthanum and oxygen, titanium is in light blue octahedra).

\subsection{Glass doping into LLTO}

As previously we talked about, the ionic conductivity of LLTO is dominated by the grain boundary, since it's always 2 to 3 orders of magnitude lower than the bulk part. One way to enhance the grain boundary ionic conductivity is doping amorphous phases into the grain boundary. Ce-Wen Nan's group introduced silica into LLTO grain boundary, the result was ionic conductivity can achieve about $10^{-4} \mathrm{~S} / \mathrm{cm}$ at room temperature [42]. $\mathrm{SiO}_{2}$ could connect lithium ions between two grain boundaries, but too much $\mathrm{SiO}_{2}$ doped in to LLTO could increase the thickness of grain boundary, thus excessive doping will negative effect on the ionic conductivity. $5 \%$ volume percent of $\mathrm{SiO}_{2}$ can make the grain boundary reach highest ionic conductivity. Before we talk about oxide glass, we have to talk about another widely used glass for conduct lithium ions, 
sulfide glasses and oxysulphide glasses. First, at room temperature, sulfide glasses can highly stable chemical react against lithium metal. Lithium ion conductivity in sulfide glasses or oxysulphide glasses system can reach $10^{-4} \mathrm{~S} / \mathrm{cm}$ or higher [43-56], which mostly higher than oxide glass and have lower activation energy, usually $0.3 \sim 0.7 \mathrm{eV}$, since it's more polarizable. In this work, we choose oxide glass system because the shortcoming of sulfide glass is its ease to hygroscopic in the air. We have to process all the experiments in argon filled glove box, include sinter with LLTO. By contrast, oxide glasses are easy to fabricate, all the process can be done in air. Lithium-borate glass system was first to be found has high lithium ionic conductivities by Otto in 1966 [57]. In this glass system, up to $\sim 25 \mathrm{~m} / \mathrm{o} \mathrm{Li}_{2} \mathrm{O}$, the activation energy, E, nearly linear decrease and electrical conductivity, $\sigma$, enhance sharply with increasing content of $\mathrm{Li}_{2} \mathrm{O}$. For the $\mathrm{Li}_{2} \mathrm{O}-\mathrm{B}_{2} \mathrm{O}_{3}$ glass system, increasing $\mathrm{SiO}_{2}$ content could decrease the glass transition temperature, which means $\mathrm{Li}_{2} \mathrm{O}-\mathrm{B}_{2} \mathrm{O}_{3}-\mathrm{SiO}_{2}$ system has more open structure. As a result, the ionic conductivity will be enhanced accompanied with the increasing non-bridging oxygen [58]. For the $\mathrm{Li}_{2} \mathrm{O}-\mathrm{SiO}_{2}$ glass system, the role of $\mathrm{B}_{2} \mathrm{O}_{3}$ is decreasing the viscosity and crystallization tendency [59]. Additionally, Masahiro Tatsumisago's group found $\mathrm{Li}_{2} \mathrm{O}-\mathrm{SiO}_{2}-\mathrm{B}_{2} \mathrm{O}_{3}$ has quite wide glass-forming region [60], as shown in figure 16. So the composition of each component of $\mathrm{Li}_{2} \mathrm{O}-\mathrm{SiO}_{2}-\mathrm{B}_{2} \mathrm{O}_{3}$ glass system is very flexible. Adding a small quantity of $\mathrm{Al}_{2} \mathrm{O}_{3}$ could enhance the crystallization temperature of the glass [61], and decreases the activation energy, which $\mathrm{B}_{2} \mathrm{O}_{3}$ increases it [62]. So in this study, we use $\mathrm{Li}_{2} \mathrm{O}-\mathrm{SiO}_{2}-\mathrm{B}_{2} \mathrm{O}_{3}-\mathrm{Al}_{2} \mathrm{O}_{3}$ system doped in to the LLTO grain boundary. The content of $\mathrm{Al}_{2} \mathrm{O}_{3}$ we used was lower than $10 \%$, because $\mathrm{Al}_{2} \mathrm{O}_{3}$ in itself is an insulator [63], too much $\mathrm{Al}_{2} \mathrm{O}_{3}$ in the oxide glass system will decrease the glass ionic conductivity. The purpose we add $\mathrm{Al}_{2} \mathrm{O}_{3}$ in the glass system is lower the activation energy and try to create lithium 


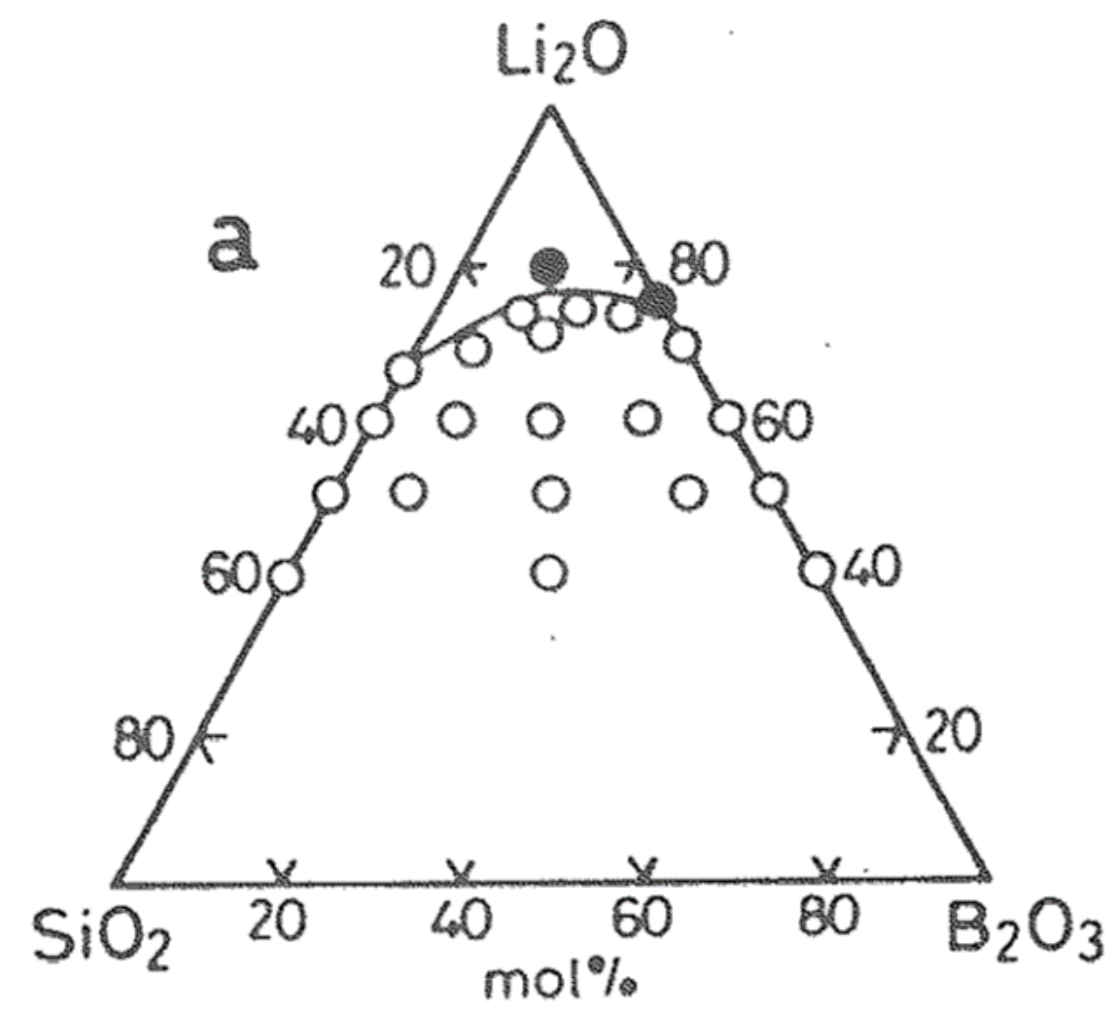

Figure 16. Glass forming region in the $\mathrm{Li}_{2} \mathrm{O}-\mathrm{SiO}_{2}-\mathrm{B}_{2} \mathrm{O}_{3}$ glass system. Open circles correspond the glassy region, closed circle correspond the crystalline region.

vacancies at our LLTO grain boundary, consequently enhance the ionic conductivity [64]. In the present work, owing to our objective of conducting lithium ion, we mixed $40 \% \mathrm{Li}_{2} \mathrm{O}$ in the glass system. Since $\mathrm{SiO}_{2}$ is the basis of glass, content of $\mathrm{SiO}_{2}$ should be higher than $\mathrm{B}_{2} \mathrm{O}_{3}$, thus we mixed (35-x)\% $\mathrm{SiO}_{2}$ and $25 \% \mathrm{~B}_{2} \mathrm{O}_{3}$. We add $\mathrm{Al}_{2} \mathrm{O}_{3}$ in this glass system for create defect at LLTO grain boundary and increase the ionic conductivity at LLTO grain boundary at the same time, so the final composition of out oxide glass is $0.4 \mathrm{Li}_{2} \mathrm{O}+0.25 \mathrm{~B}_{2} \mathrm{O}_{3}+(0.35-\mathrm{x}) \mathrm{SiO}_{2}+\mathrm{xAl}_{2} \mathrm{O}_{3}$, $\mathrm{x}=1 \%, 5 \%, 10 \%$ respectly.

1.5 Objective of this work 
To achieve the best ionic conductivity performance for LLTO, there are several conditions we need to approach for the doping glass:

(1) Synthesize ordered lanthanum and single phase LLTO.

As previously mentioned, to reach the highest ionic conductivity of LLTO, it is necessary to find ways to synthesis lanthanum ordered and single phase LLTO. Multiple phase structures coexist in one piece of sample could hinder the lithium ions movement. In this work, we synthesized single phase tetragonal perovskite LLTO, used furnace cooled method to make sure lanthanum in LLTO is ordered and only one phase exists in our sample. Although lithium ions in quenched cooling LLTO can migrate in three directions, the structure is not stable and multiple phases coexist in one piece of the sample. Chaotic atom arrangement in LLTO only can enhance the activation energy.

(2) Develop oxide glass which lithium ion conductivity higher than $10^{-3} \mathrm{~S} / \mathrm{cm}$.

The grain bulk ionic conductivity of LLTO is around $10^{-3} \mathrm{~S} / \mathrm{cm}$ and ionic conductivity at grain boundary dominate the entire value, so the glass we doped into LLTO grain boundary should equal or higher and the LLTO grain bulk one.

(3) Chemical reaction stable with lithium.

We just have to admit that not all kinds of oxide glasses can good match with LLTO, though composition range for oxide glass is flexible. Thus, we should consider the crystallization of the oxide glass. Glasses which easy to crystallize are not good for conduction lithium ion and match with oxide electrolyte. Lithium ions have to go through the grain boundary. When lithium ions move into LLTO, we have to make sure electronic conductivity, as we talked about electrochemical lithium insert into $\mathrm{LaTiO}_{3}$ previously, not increase, or short circuit will happen for the entire circuit. 
(4) Work effectively under $200^{\circ} \mathrm{C}$.

In practical application, solid state electrolyte sometimes need to work under extreme temperature. So we request glasses not melted and keep the magnitude of ionic conductivity at high temperature, usually under $200{ }^{\circ} \mathrm{C}$ is enough. 


\section{Chapter 2: Experimental methods}

\subsection{Synthesis of LLTO}

LLTO powder was synthesized by traditional solid state reaction method. The starting materials were $\mathrm{Li}_{2} \mathrm{CO}_{3}, \mathrm{La}_{2} \mathrm{O}_{3}$ and $\mathrm{TiO}_{2}$ with a molar ratio of $\mathrm{Li}: \mathrm{La}: \mathrm{Ti}=0.4: 0.6: 1$. In consideration of lithium evaporation during high temperature sintering, we added a stoichiometric excess of $20 \%$ more $\mathrm{Li}_{2} \mathrm{CO}_{3}$ into the starting materials. After 24 hours ball milling in ethanol and then drying in an oven, the starting materials powder were sintered at $800^{\circ} \mathrm{C}$ for 4 hours for decarbonization, and then sintered at $1150^{\circ} \mathrm{C}$ for 12 hours. This material was grounded and then re-sintered for a total of three sintering cycles. For LLTO pellet, the synthesis method is almost same as LLTO powder, the only different is the final step sinter. After 2 cycles sinter under $1150^{\circ} \mathrm{C}$ for 12 hours with intermediate ground, the LLTO powder was pressed into pellet under $33362 \mathrm{~N}$, three minutes and three times. Then the pellet was sintered under $1300^{\circ} \mathrm{C}$ for 12 hours. Lithium evaporation during high temperature sintering is a serious problem [65]. But for this material, especially for the pellet sinter, under lower temperature cannot make the pellet dense enough [66-70], which will significantly decrease the ionic conductivity [71]. Furthermore, increase the sinter temperature can also increase the LLTO grain size, and the ionic conductivity enhance as the grain size enlarge.

\subsection{Glass fabrication}

The oxide glass was made via melt-quench method in the air. The starting chemicals were $\mathrm{Li}_{2} \mathrm{O}, \mathrm{B}_{2} \mathrm{O}_{3}, \mathrm{SiO}_{2}$ and $\mathrm{Al}_{2} \mathrm{O}_{3}$ with composition 0.4:0.25:0.35-x:x, in which $\mathrm{x}=0,0.05$ and 0.1 . Chemicals for fabricating glass also mixed for 24 hours in ethanol and drying in a oven. After 
drying in a oven, the starting materials powder was melted at $1050^{\circ} \mathrm{C}$ in a crucible for three hours in the furnace, and then took out and quenched on a cold metal plate.

\subsection{Glass doping into LLTO}

First we grounded glass from chunk into powder. Then $1 \%, 2 \%, 3 \%, 5 \%$ weight percent glass powder for all the compositions, as we described above, were separately mixed with LLTO powder. Ball milling the glass powder and LLTO powder in ethanol for 24 hours, drying in oven, press the powder into pellet and sinter at $1300^{\circ} \mathrm{C}$ for 8 hours.

\subsection{Charactristics}

The crystalline structure of the LLTO and LLTO/oxide glass were characterized by PANalytical XRD with a Cu-ka1 $8047.2 \mathrm{eV}$ source and JEOL-2100 TEM under 200kv beam voltage. Side-entry double tilt holder was used to turn the grains we observed in low index. TEM specimen was polished by Allied High Tech's MultiPrep Polisher. After polishing the LLTO pellet to 20 micros, ion mill was used to mill the LLTO thin slice. The LLTO/oxide glass cross sectional microstructure was observed by Hitachi S-4700F Scanning Electron Microscope. For the ionic conductivity measurement, we sputtered Au on both sides of the sample surface and painted silver paste on the surfaces which were connected to a silver current collector wire. We applied alternating circuit (AC) impedance spectroscopy by Metrohm Autolab b.v. to test the electrical conductivity in the temperature range from $30^{\circ} \mathrm{C}$ to $150^{\circ} \mathrm{C}$. The density of the LLTO/oxide glass pellets were measured by the Archimedes method. 


\section{Chapter 3: Synthesis and characteristics of LLTO electrolyte}

\subsection{Structural characteristics of LLTO electrolytes}

The XRD pattern of the LLTO is presented in Figure 17. The structure of LLTO can be labeled as perovskite $(\mathrm{Pm}-3 \mathrm{~m})$ with lattice parameter $3.871 \AA$ and only one phase was detected, so we successfully synthesized pure LLTO.

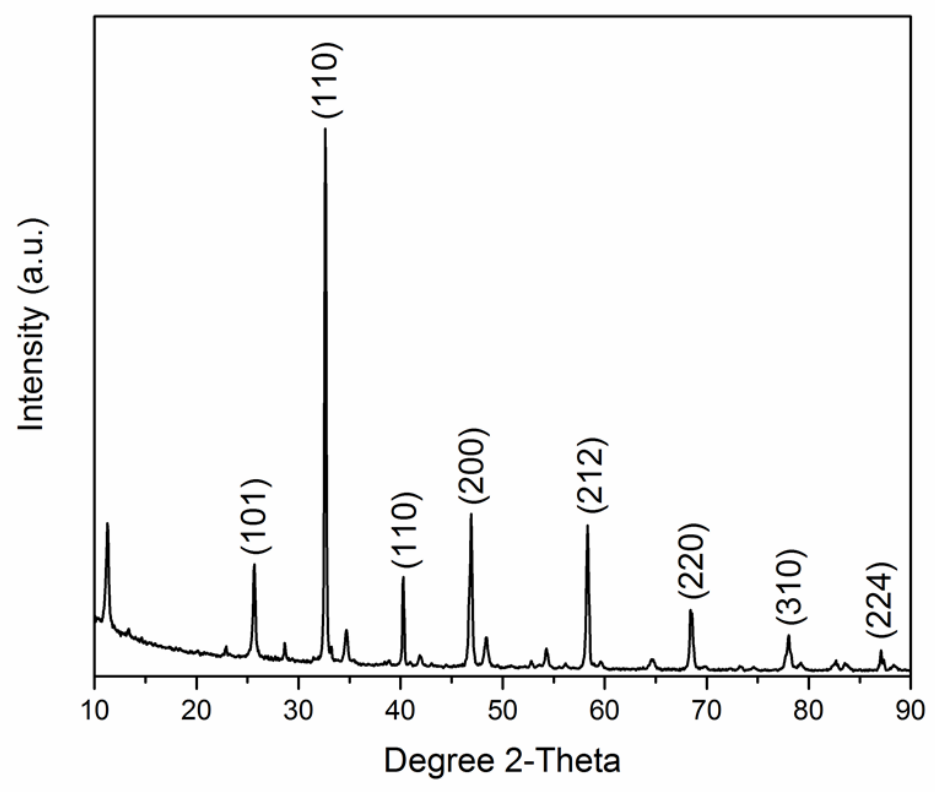

Figure 17. X-ray diffraction spectra of LLTO.

Our TEM result coincide with the XRD result. Figure 18 shows the high-resolution TEM graph of LLTO and the corresponding selected area electron diffraction which scattered from [010] direction. Owing to the poor value of the lithium ion scattering factor in practice, we assume that lanthanum ion LLTO build the frame of the perovskite structure. 
From Figure 18, we can clearly see the right-angle grain boundary of LLTO, this is a typical structural characteristic of LLTO $[25,26,36,51]$. The extra diffraction spots between transmission spot and (100) or (001) indicate single perovskite supercells exist in this LLTO structure, $a$ and $c$ parameters are doubled at this point of view. We can also see bright layers and dark layer are alternated appear in our high resolution TEM graph. This is due to the variation lanthanum alternate layers. Bright layers are the lanthanum rich layers and the dark layers are the lanthanum deficient layers [29, 37, 54].

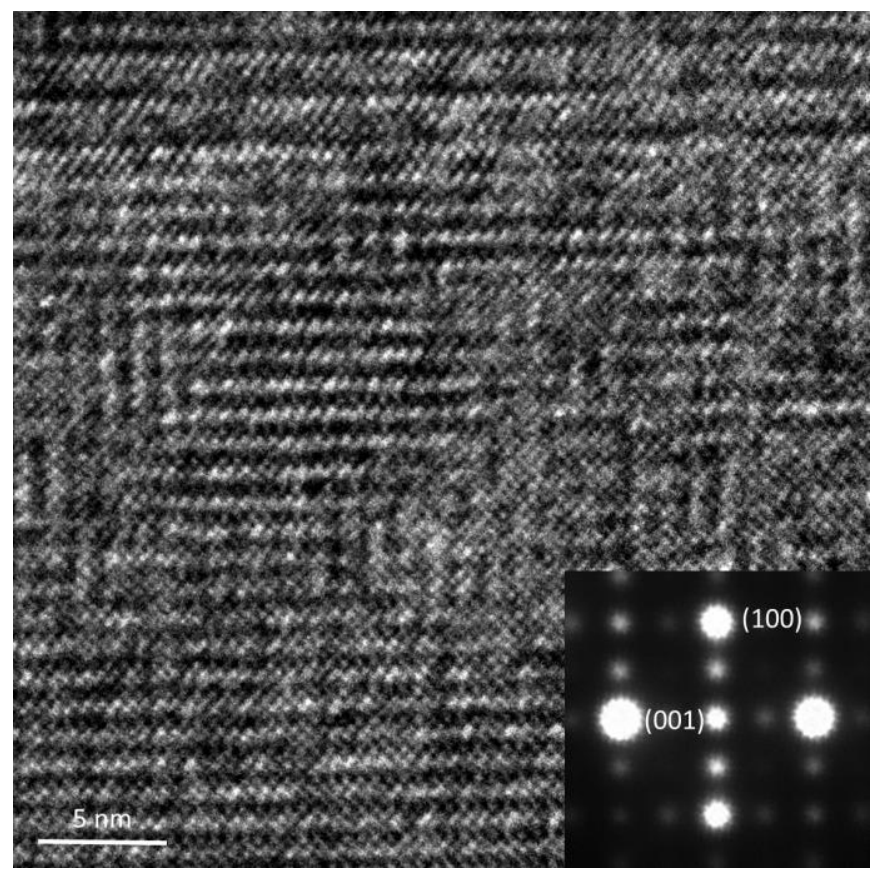

Figure 18. High resolution TEM graph of LLTO with selected area diffraction.

3.2 Examinations of lithium ions in LLTO electolytes

As previously we mentioned, since the scatter factor of lithium ion is really small in practical use, and it cannot be identified by the selected area electron diffraction, it's difficult to determine the precise positons of lithium ions in LLTO perovskite structure. Xiang Gao's group used the 
direct annular bright-field (ABF) to observe lithium ions locations in LLTO. From their result, lithium ions may locate in O4 windows in lithium poor LLTO and may locate close to A-site in lithium rich LLTO [37]. Neutron diffraction was widely used to study the locations of the lithium ions in LLTO $[32,55,56]$. Most studies believed that lithium ions are located in the interstitial sites in LLTO as we mentioned in chapter 1. Figure 19 shows some typical LLTO structures, data from literatures of neutron diffraction study of LLTO.
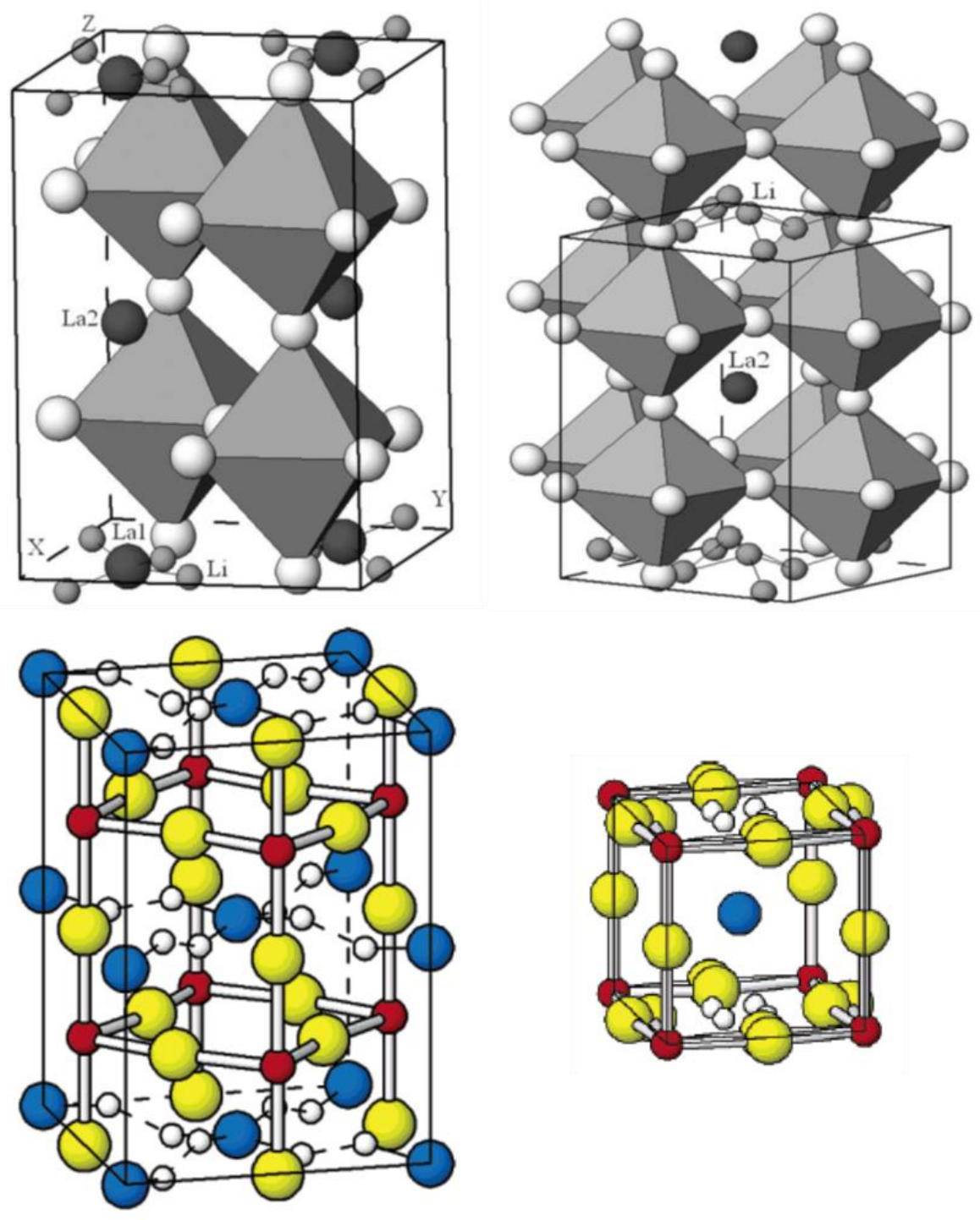

Figure 19. Crystal structure of LLTO built from neutron diffraction data. 


\subsection{Ionic conductivity test of LLTO}

Among all of the solid state electrolyte candidates for lithium battery applications, the perovskite structure lanthanum lithium titanate $\left(\mathrm{La}_{2 / 3-x} \square_{1 / 3-2 x} \mathrm{Li}_{3 x} \mathrm{TiO}_{3}\right)$ has the best lithium ionic conductivity, which can reach $10^{-3} / \mathrm{cm}$ in the grain bulk, but typically only $10^{-6} \mathrm{~S} / \mathrm{cm}$ at the grain boundary [2]. Figure 20 shows the SEM graph of the LLTO cross section.

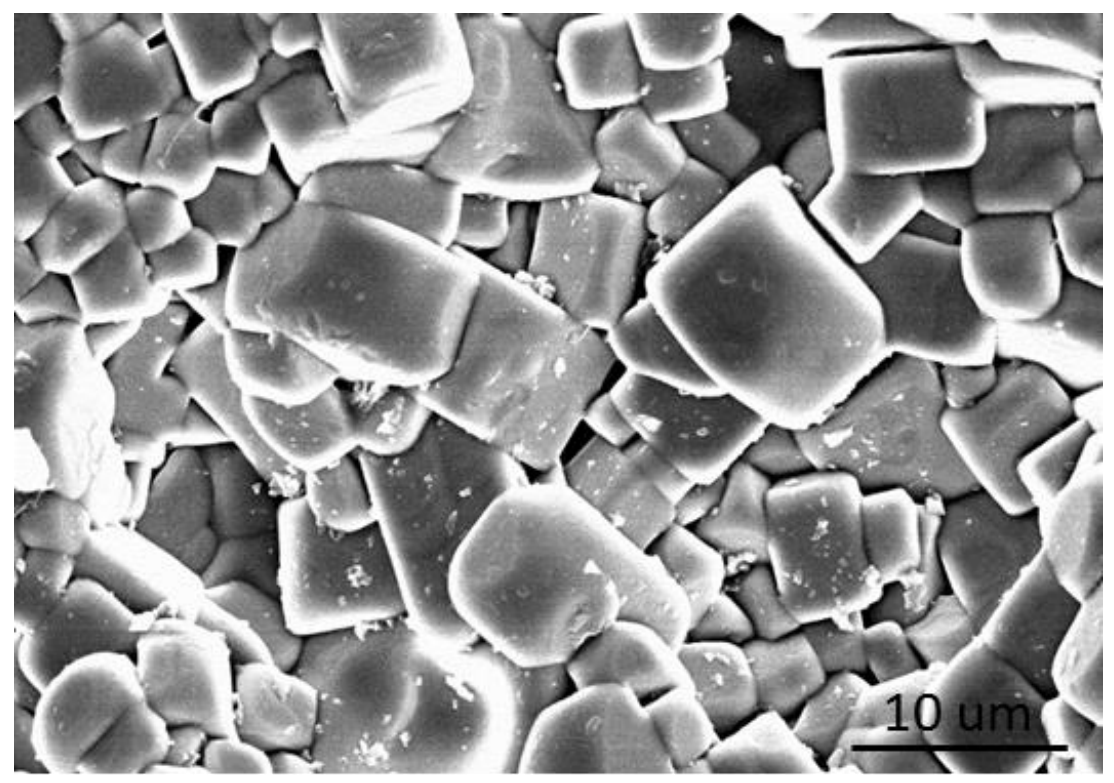

Figure 20. Typical SEM micrograph of LLTO cross section.

The SEM graph confirmed the LLTO pellets we synthesized is dense enough, the LLTO grains are tightly compacted to each other. This is really important for LLTO ionic conductivity, because if gaps exist between LLTO grains, the lithium ions will be stuck at grain boundaries and leads to enormous decrease of ionic conductivity. The density of LLTO we synthesized is related $97.7 \%$ to theoretical density. Figure 20 shows a typical Nyquist plot of the LLTO solid state 
electrolyte with Au sputtered blocking electrodes. The typical plot measured by electrochemical impedance includes a semicircle at high and medium frequencies, corresponding to the ionic conductivity in the LLTO grain bulk and at the grain boundary, and a straight line at low frequency which presents the block effect of Au. We used Z-View software to analyze the electrochemical impedance data, and calculated ion conductivity by equation (4):

$\sigma=\frac{l}{A R}$

where $l$ is the thickness of the sample pellets, $A$ is the sample surface area and $R$ is the sample resistant obtained by fitting the semicircle at high frequency.

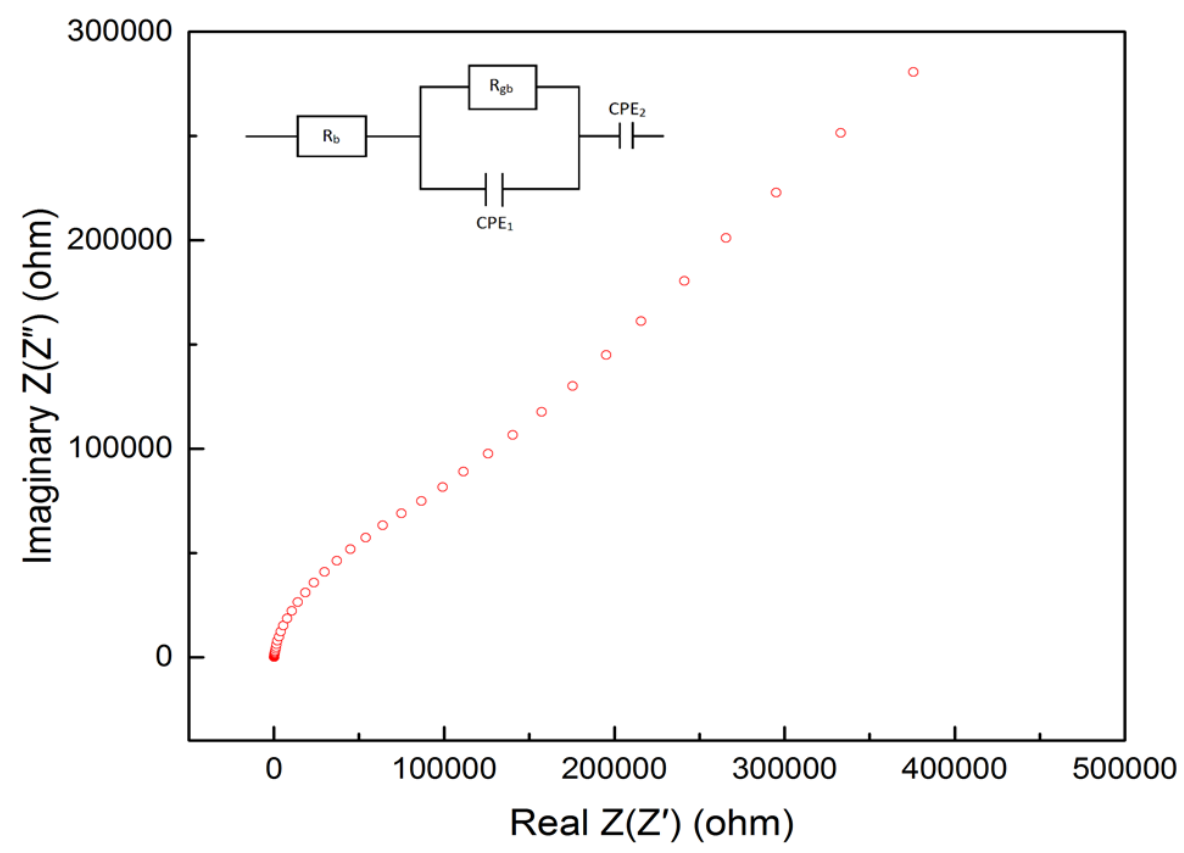

Figure 20. Typical electrochemical impedance plot of LLTO at $30^{\circ} \mathrm{C}$ with equivalent circuit inset. 
Figure 21 shows the LLTO ionic conductivity in the grain bulk and at the grain boundary as the temperature increases. For ceramic electrolyte, ions move through the point defect sites and temperature corresponding to the energy of the ions movement, so as the temperature increase, the ionic conductivity enhanced, especially in the grain bulk. For LLTO grain bulk, we found that ionic conductivity is $1.12 \times 10^{-3} \mathrm{~S} / \mathrm{cm}$ at $30^{\circ} \mathrm{C}$ and it can reach $1.63 \times 10^{-2} \mathrm{~S} / \mathrm{cm}$ at $150^{\circ} \mathrm{C}$. The ionic conductivity at the grain boundary is $5.61 \times 10^{-5} \mathrm{~S} / \mathrm{cm}$ and $1.64 \times 10^{-3} \mathrm{~S} / \mathrm{cm}$ at $30^{\circ} \mathrm{C}$ and $150^{\circ} \mathrm{C}$, respectively. As the temperature increase, the activation energy of LLTO decrease. The decreasing activation energy enhance the ionic conductivity.

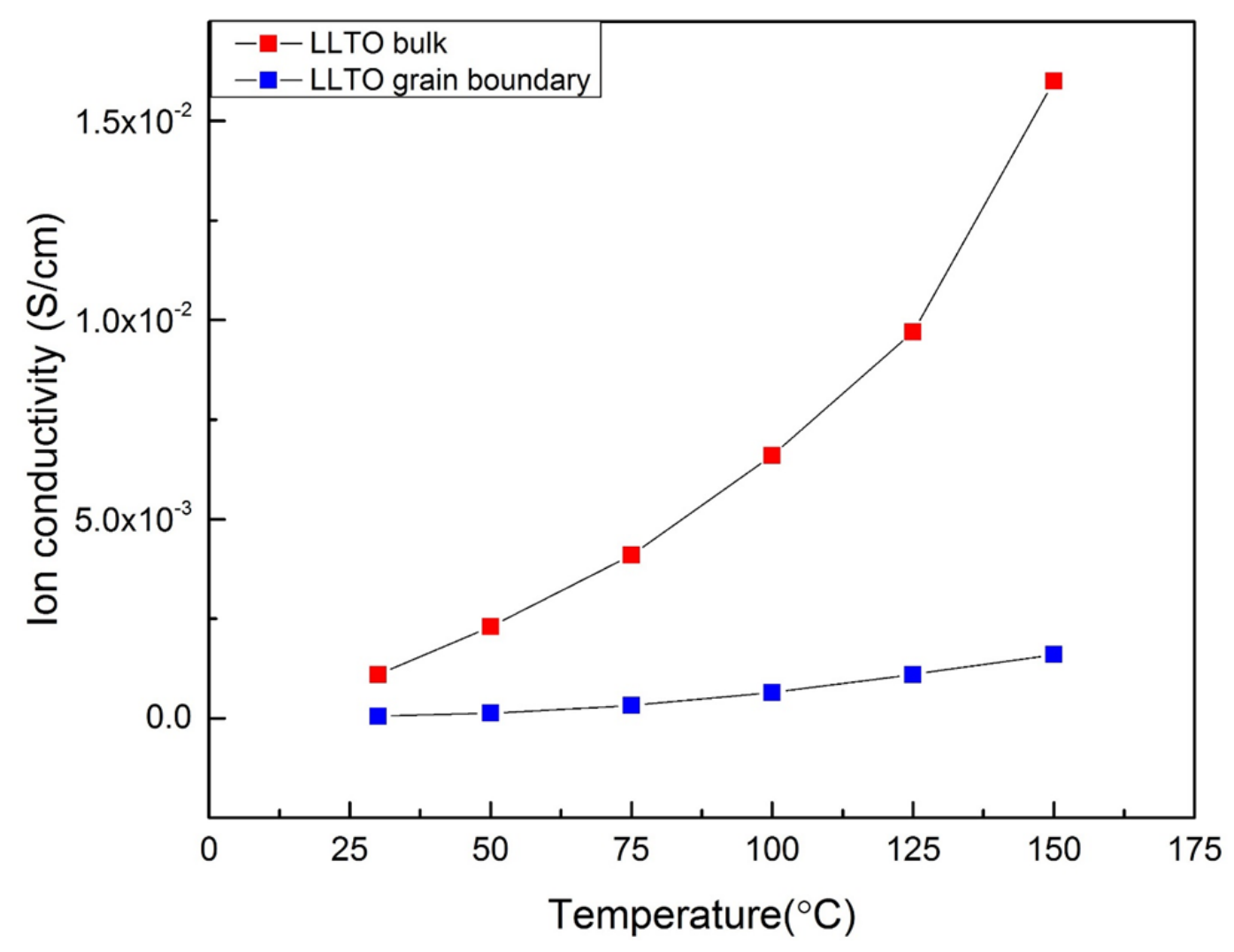

Figure 21. LLTO grain bulk and grain boundary ion conductivity under different temperature. 


\section{Chapter 4: LLTO electrolytes doped with oxide glasses}

It was noted that doping an amorphous phase into the LLTO grain boundary can substantially increase its ionic conductivity $[42,63,72,73]$. We fabricated glass with a composition of $0.4 \mathrm{Li}_{2} \mathrm{O}+0.25 \mathrm{~B}_{2} \mathrm{O}_{3}+(0.35-\mathrm{x}) \mathrm{SiO}_{2}+\mathrm{xAl}_{2} \mathrm{O}_{3}$ where $\mathrm{x}=0,0.05,0.1$, and the weight percentages of the glasses added into LLTO are 1\%, 2\%, 3\%, 5\%. The XRD patterns of the pure LLTO and LLTO dope different oxide glass composition and different weight percent are presented in Figure 22. The structure of LLTO can be labeled as perovskite (Pm-3m) and only one phase was detected. The diffraction peaks of LLTO with various amounts and compositions of oxide glass are almost the same and were also found to exhibit only one crystalline phase. So, the oxide glass can only be considered exist at the LLTO grain boundary and can be seen as an amorphous phase in the electrolyte. Regardless of the glass composition, on crystalline form for this glass.

Typical SEM micrographs of the LLTO/oxide glass pellet cross section are shown in Figure 23. Different oxide glass compositions are found to have no effect on the LLTO grain size. However, a varying particulate pattern can be seen to arise at the surface of the LLTO grain as the amount of $\mathrm{Al}_{2} \mathrm{O}_{3}$ increases. Although the largest amount of the added $\mathrm{Al}_{2} \mathrm{O}_{3}$ component is not enough to cover the LLTO grain and separate out from the glass system, it was found that larger quantities of $\mathrm{Al}_{2} \mathrm{O}_{3}$ have a negative effect on the ionic conductivity at the grain boundary. From

figure 23 (c), we can clearly see white particles separated out, because $10 \%$ weight percent of $\mathrm{Al}_{2} \mathrm{O}_{3}$ is already too much in the oxide glass system. $\mathrm{Al}_{2} \mathrm{O}_{3}$ is insulating material, too much $\mathrm{Al}_{2} \mathrm{O}_{3}$ adds into glass will decrease the ionic conductivity. 
Figure 24 is a typical TEM graph of LLTO grain boundary. From figure 24, glass sufficiently filled between LLTO grains can be seen which we cannot clearly observed by SEM. Glass fully filled into LLTO grain boundary is crucial for ion conduct at grain boundary.

The ionic conductivity of LLTO doped different composition of $\left(0.4 \mathrm{Li}_{2} \mathrm{O}+0.25 \mathrm{~B}_{2} \mathrm{O}_{3}+(0.35-\right.$ x) $\mathrm{SiO}_{2}+\mathrm{xAl}_{2} \mathrm{O}_{3}$ ) oxide glasses with different weight percent are shown in Figure 25. From Figure 25(a) (c) (e), we can clearly see that an increasing temperature enhance the ionic conductivity in the LLTO grain bulk. But at the same temperature and same composition of glass, the ion conductivities of LLTO grain bulk with different weight percent of amorphous phases are almost the same. Figure 25(b) shows the grain boundary ionic conductivity of $\mathrm{LLTO} /\left(0.4 \mathrm{Li}_{2} \mathrm{O}+0.25 \mathrm{~B}_{2} \mathrm{O}_{3}+0.35 \mathrm{SiO}_{2}\right)$. In this glass composition doped in to LLTO, $3 \mathrm{wt} \%$ amorphous phase doped into LLTO offers the best ionic conductivity. Additional weight percent of glass doped into the LLTO grain boundary leads to a decreasing for the ionic conductivity. Figure $25(\mathrm{~d})$ shows the ionic conductivity at the grain boundary of LLTO with $\left(0.4 \mathrm{Li}_{2} \mathrm{O}+0.25 \mathrm{~B}_{2} \mathrm{O}_{3}\right.$ $\left.+0.3 \mathrm{SiO}_{2}+0.05 \mathrm{Al}_{2} \mathrm{O}_{3}\right)$ amorphous phase in different weight percentage. This time, under any temperature we tested, $2 \mathrm{wt} \%\left(0.4 \mathrm{Li}_{2} \mathrm{O}+0.25 \mathrm{~B}_{2} \mathrm{O}_{3}+0.3 \mathrm{SiO}_{2}+0.05 \mathrm{Al}_{2} \mathrm{O}_{3}\right)$ doped into LLTO reaches the highest ion conductivity. Grain boundary ionic conductivities of LLTO doped with $\left(0.4 \mathrm{Li}_{2} \mathrm{O}+0.25 \mathrm{~B}_{2} \mathrm{O}_{3}+0.25 \mathrm{SiO}_{2}+0.1 \mathrm{Al}_{2} \mathrm{O}_{3}\right)$ composition is illustrated in Figure 25(f). The ionic conductivity decrease as the weight percentage of oxide glass increasees. This demonstrates the detrimental effect of a surplus amount of aluminum oxide in our glass system.

Densities of LLTO/oxide glass pellets are presented in Table 2. For both compositions of the pellets, $2 \mathrm{wt} \%$ oxide glass doped samples have the highest density. From the density data, we can surmise that an approximate amount of $2 \mathrm{wt} \%$ amorphous phase is the optimum amount to fill the LLTO grain boundary. As we mentioned before, $\mathrm{LLTO}+(0.4 \mathrm{Li} 2 \mathrm{O}+0.25 \mathrm{~B} 2 \mathrm{O} 3+0.35 \mathrm{SiO} 2)$ reaches 

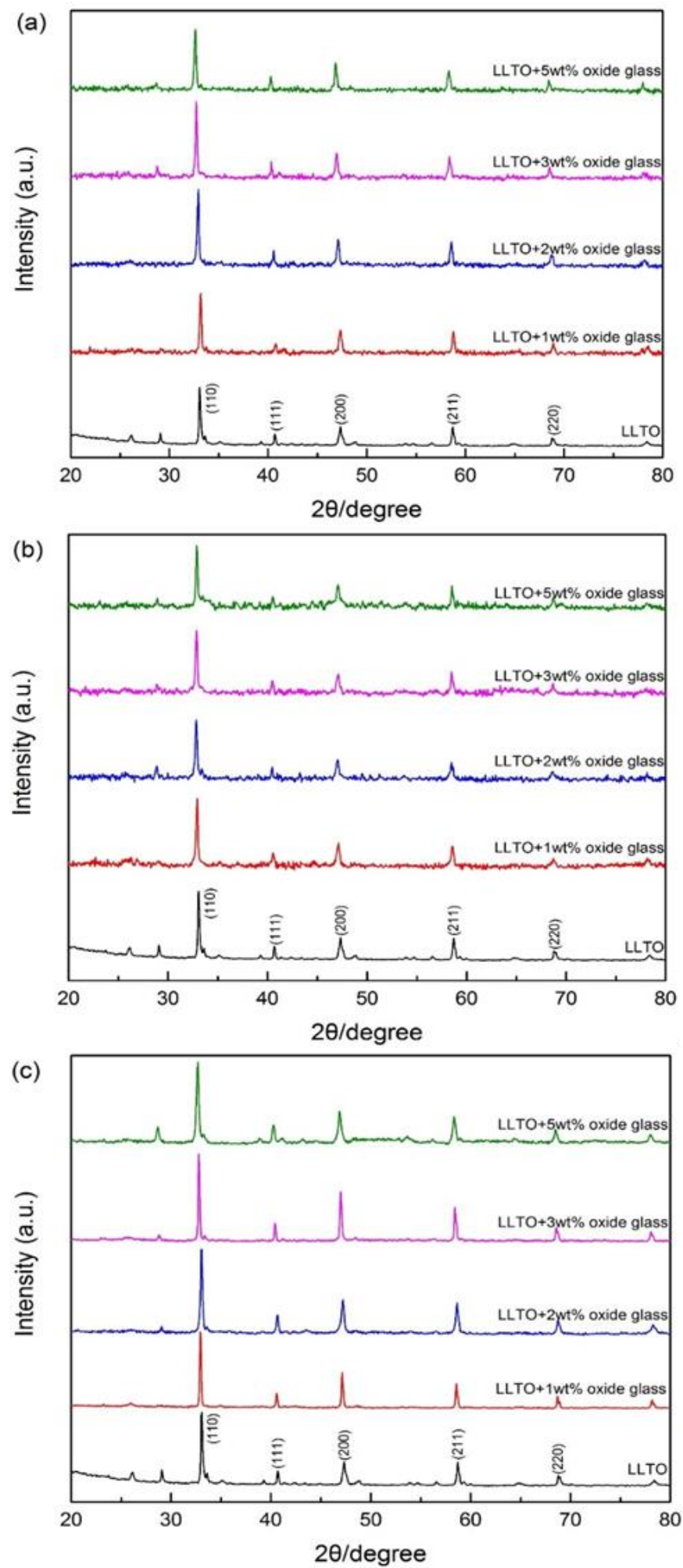

Figure 22. X-ray diffraction spectra of LLTO. (a) LLTO+(0.4 $\left.\mathrm{Li}_{2} \mathrm{O}+0.25 \mathrm{~B}_{2} \mathrm{O}_{3}+0.35 \mathrm{SiO}_{2}\right)$, (b) $\mathrm{LLTO}+\left(0.4 \mathrm{Li}_{2} \mathrm{O}+0.25 \mathrm{~B}_{2} \mathrm{O}_{3}+0.3 \mathrm{SiO}_{2}+0.05 \mathrm{Al}_{2} \mathrm{O}_{3}\right)$ and (c) $\mathrm{LLTO}+\left(0.4 \mathrm{Li}_{2} \mathrm{O}+0.25 \mathrm{~B}_{2} \mathrm{O}_{3}+0.25 \mathrm{SiO}_{2}+0.1 \mathrm{Al}_{2} \mathrm{O}_{3}\right)$. 


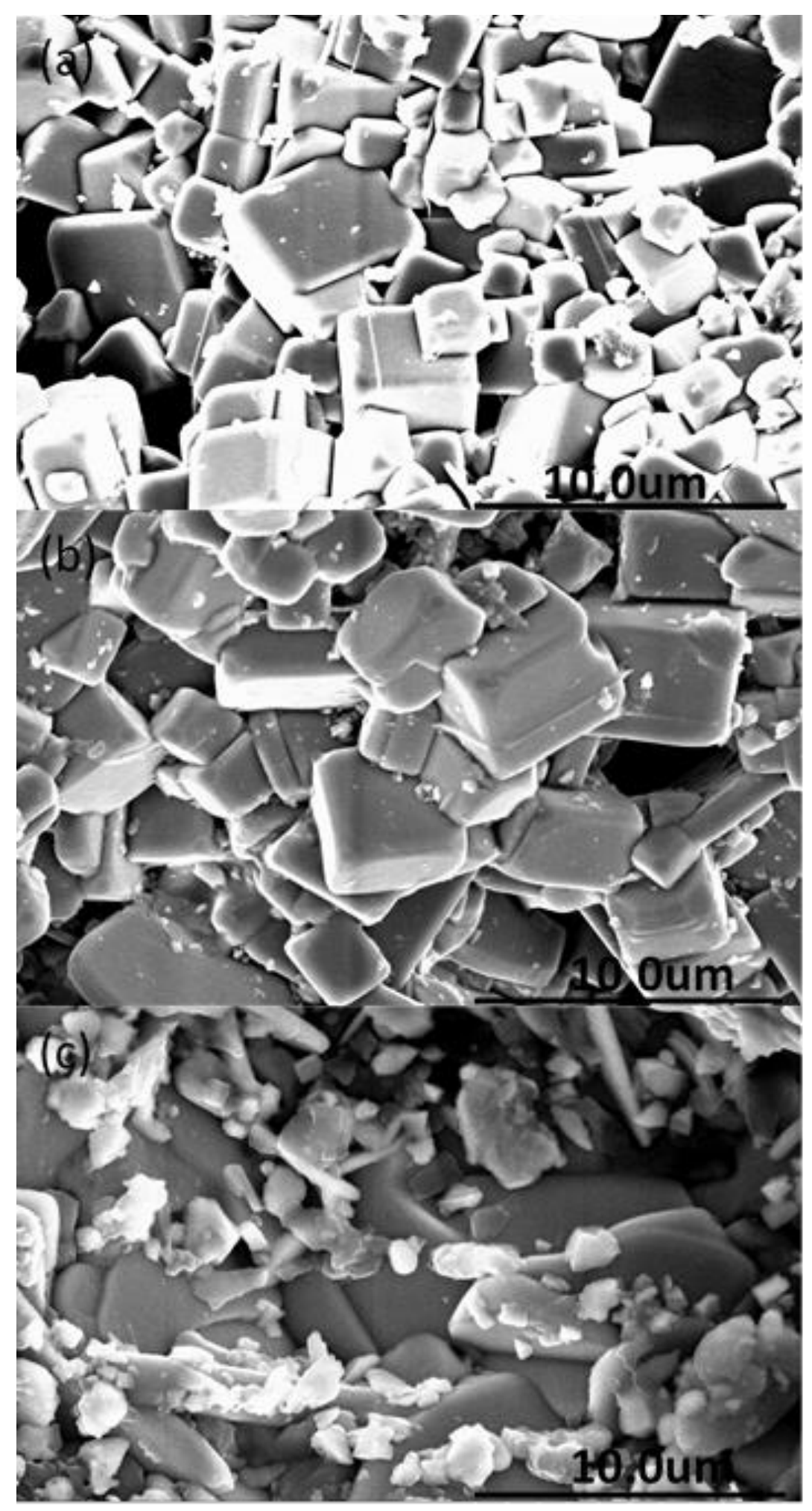

Figure 23. Typical SEM micrographs of (a) LLTO $+5 \mathrm{wt} \%\left(0.4 \mathrm{Li}_{2} \mathrm{O}+0.25 \mathrm{~B}_{2} \mathrm{O}_{3}+0.35 \mathrm{SiO}_{2}\right)$, (b) LLTO $+5 \mathrm{wt} \%\left(0.4 \mathrm{Li}_{2} \mathrm{O}+0.25 \mathrm{~B}_{2} \mathrm{O}_{3}+0.3 \mathrm{SiO}_{2}+0.05 \mathrm{Al}_{2} \mathrm{O}_{3}\right)$ and (c) $\mathrm{LLTO}+5 \mathrm{wt} \%$ $\left(0.4 \mathrm{Li}_{2} \mathrm{O}+0.25 \mathrm{~B}_{2} \mathrm{O}_{3}+0.25 \mathrm{SiO}_{2}+0.1 \mathrm{Al}_{2} \mathrm{O}_{3}\right)$. 


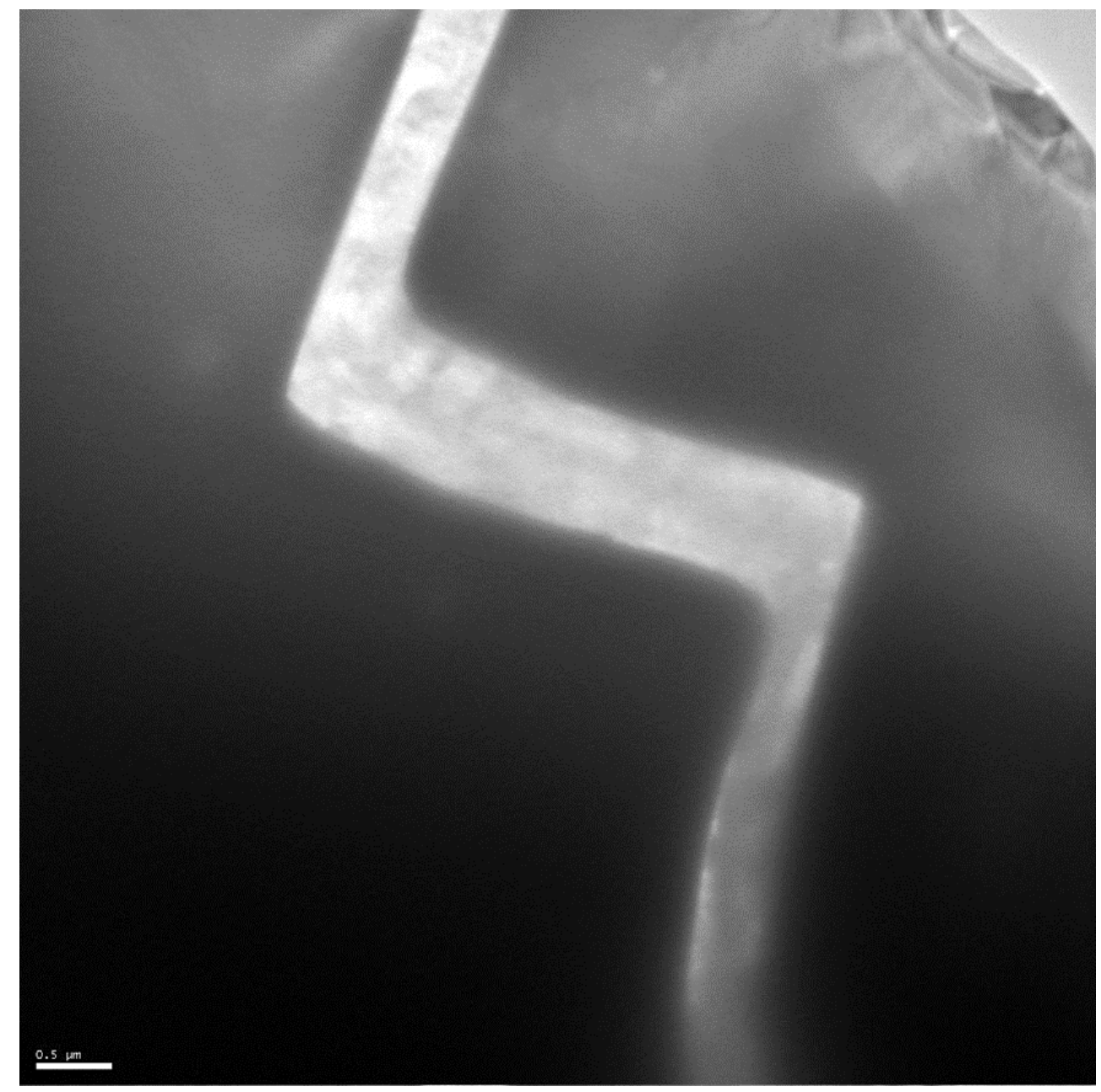

Figure 24. TEM graph of amorphous phase doped into LLTO grain boundary.

best grain ionic conductivity and that the $3 \mathrm{wt} \%$ amorphous phased doped LLTO plus $2 \mathrm{wt} \%(0.4 \mathrm{Li} 2 \mathrm{O}+0.25 \mathrm{~B} 2 \mathrm{O} 3+0.3 \mathrm{SiO} 2+0.05 \mathrm{Al} 2 \mathrm{O} 3)$ gives the highest grain boundary ionic conductivity. More weight percent of oxide glass doping into LLTO is redundant. Since the density of glass is lower than LLTO, excess $2 \%$ weight percent glass doped into LLTO reduce the enitre density. 
Effect of adding alumina

From figure 25 (b) and the densities of sample pellets, we see that, although $3 \mathrm{wt} \%$ amorphous phase doped into LLTO is a little bit excess, it is not harmful for the grain boundary ionic conductivity. The composition proportion of $\mathrm{Li}_{2} \mathrm{O}$ and $\mathrm{B}_{2} \mathrm{O}_{3}$ are fixed, we only changed $\mathrm{SiO}_{2} / \mathrm{Al}_{2} \mathrm{O}_{3}$ ratio. Increasing the $\mathrm{Al}_{2} \mathrm{O}_{3}$ component reduces the $\mathrm{SiO}_{2}$ component and decreases $\mathrm{SiO}_{2} / \mathrm{B}_{2} \mathrm{O}_{3}$ ratio. In this way, we can decrease the $\mathrm{Li}_{2} \mathrm{O}-\mathrm{B}_{2} \mathrm{O}_{3}-\mathrm{SiO}_{2}$ glass crystallization tendency [58], although the ternary glass ionic conductivity slightly increases with the increase of $\mathrm{SiO}_{2} / \mathrm{B}_{2} \mathrm{O}_{3}$ ratio. The broader the spectrum of glass formers, the easier we can achieve an amorphous phase, which is significant for the lithium ion isotropic conductivity at the grain boundary. Furthermore, crystallization in oxide glass can leads to electrical conductivity [61] which must be avoided for the electrolyte applications. On the glass formation aspect, the role of $\mathrm{Al}_{2} \mathrm{O}_{3}$ can act as a network former and modifier. The trivalent $\mathrm{Al}^{+3}$ also enhances the free lithium ion concentration, because of the electric neutrality of the glass system [17]. C.C. Liang and A.C. Khandkar mentioned that $\mathrm{Li}^{+}$vacancies can be created after the dissolution of $\mathrm{Al}_{2} \mathrm{O}_{3}$ into $\mathrm{LiI}$ at the interface $[74,75]$. The same mechanism can also explain our work. Equation (5) elucidates how the $\mathrm{Li}^{+}$vacancies are generated in our glass system.

$$
2 \mathrm{Li}_{2} \mathrm{O} \stackrel{\mathrm{Al}_{2} \mathrm{O}_{3}}{\longrightarrow} 2 \mathrm{Al} \mathrm{L}_{L i}+2 V_{L i}^{\prime}+\mathrm{O}_{i}^{\prime \prime}+3 \mathrm{O}_{O}^{x}
$$



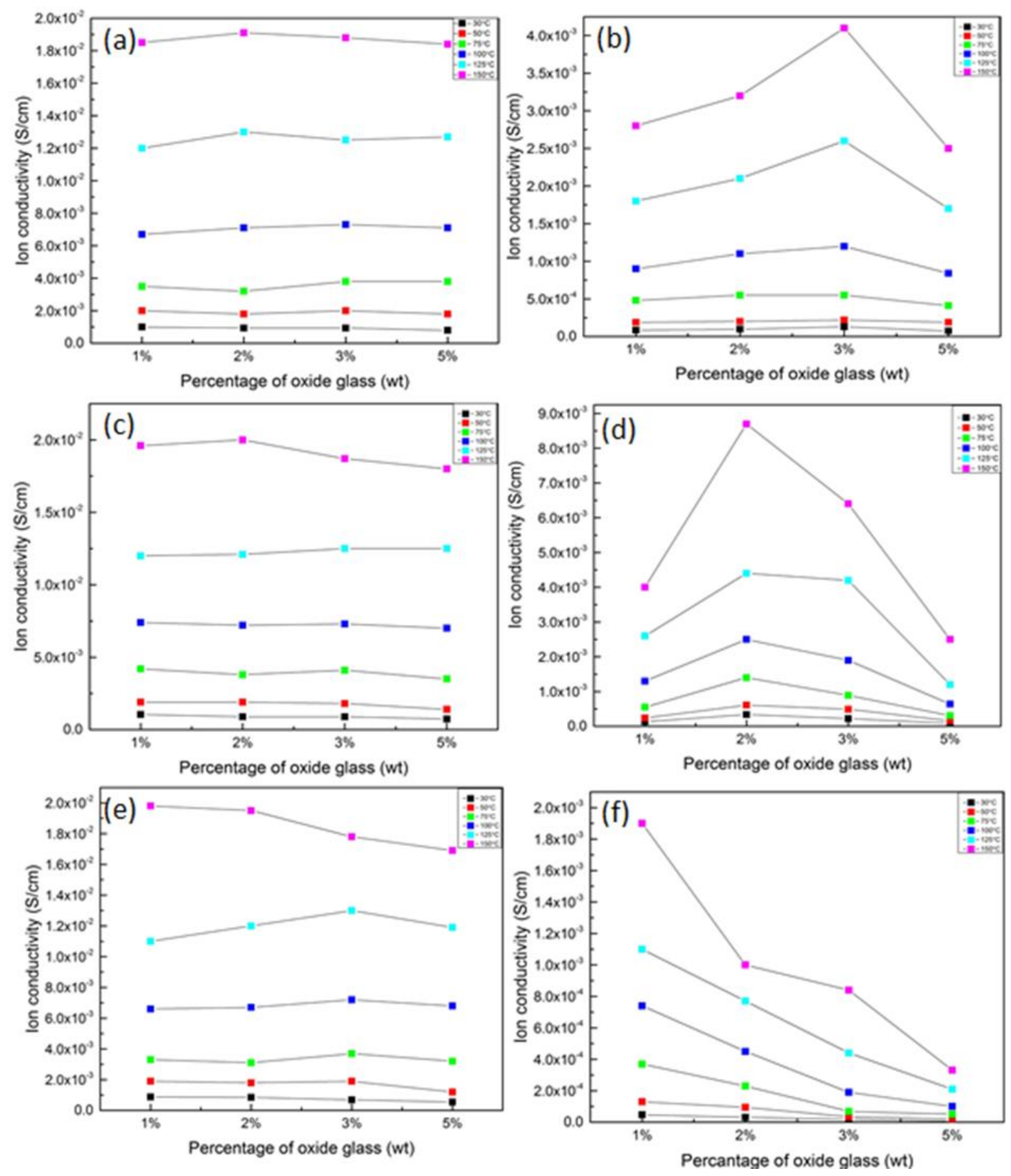

Figure 25. (a) $\mathrm{LLTO}+\left(0.4 \mathrm{Li}_{2} \mathrm{O}+0.25 \mathrm{~B}_{2} \mathrm{O}_{3}+0.35 \mathrm{SiO}_{2}\right)$ grain bulk ion conductivity. (b)

$\mathrm{LLTO}+\left(0.4 \mathrm{Li}_{2} \mathrm{O}+0.25 \mathrm{~B}_{2} \mathrm{O}_{3}+0.35 \mathrm{SiO}_{2}\right)$ grain boundary ion conductivity. (c)

LLTO $+\left(0.4 \mathrm{Li}_{2} \mathrm{O}+0.25 \mathrm{~B}_{2} \mathrm{O}_{3}+0.3 \mathrm{SiO}_{2}+0.05 \mathrm{Al}_{2} \mathrm{O}_{3}\right)$ grain bulk ion conductivity. (d)

LLTO $+\left(0.4 \mathrm{Li}_{2} \mathrm{O}+0.25 \mathrm{~B}_{2} \mathrm{O}_{3}+0.3 \mathrm{SiO}_{2}+0.05 \mathrm{Al}_{2} \mathrm{O}_{3}\right)$ grain boundary ion conductivity. (e)

$\mathrm{LLTO}+\left(0.4 \mathrm{Li}_{2} \mathrm{O}+0.25 \mathrm{~B}_{2} \mathrm{O}_{3}+0.25 \mathrm{SiO}_{2}+0.1 \mathrm{Al}_{2} \mathrm{O}_{3}\right)$ grain bulk ion conductivity. (f)

$\mathrm{LLTO}+\left(0.4 \mathrm{Li}_{2} \mathrm{O}+0.25 \mathrm{~B}_{2} \mathrm{O}_{3}+0.25 \mathrm{SiO}_{2}+0.1 \mathrm{Al}_{2} \mathrm{O}_{3}\right)$ grain boundary ion conductivity. 
Table 2. LLTO/oxide glass pellets density

\begin{tabular}{l|llll}
\hline & $1 w t \%$ & $2 w t \%$ & $3 w t \%$ & $5 w t \%$ \\
\hline LLTO+(0.4Li $\left.2 \mathrm{O}+0.25 \mathrm{~B}_{2} \mathrm{O}_{3}+0.35 \mathrm{SiO}_{2}\right)$ & $4.65 \mathrm{~g} / \mathrm{cm}^{3}$ & $4.81 \mathrm{~g} / \mathrm{cm}^{3}$ & $4.60 \mathrm{~g} / \mathrm{cm}^{3}$ & $4.56 \mathrm{~g} / \mathrm{cm}^{3}$ \\
& & & & \\
LLTO+(0.4Li $\left.2 \mathrm{O}+0.25 \mathrm{~B}_{2} \mathrm{O}_{3}+0.3 \mathrm{SiO}_{2}+0.05 \mathrm{Al}_{2} \mathrm{O}_{3}\right)$ & $4.67 \mathrm{~g} / \mathrm{cm}^{3}$ & $4.83 \mathrm{~g} / \mathrm{cm}^{3}$ & $4.73 \mathrm{~g} / \mathrm{cm}^{3}$ & $4.58 \mathrm{~g} / \mathrm{cm}^{3}$ \\
\hline
\end{tabular}

As shown in Figure 25 (b) (d) (f), additional alumina added into the glass will lead to an ionic conductivity decrease due to the already existing $\mathrm{Li}^{+}$defects becoming occupied by $\mathrm{Al}^{+}$, so lithium ions penetrate through LLTO grain boundary was obstruct. Additionally, the ionic conductivity will be decreased as the percentage of the $\mathrm{SiO}_{2}$ component is reduced [58]. 


\section{Chapter 5: Conclusions}

$\mathrm{La}_{2 / 3-x} \square_{1 / 3-2 x} \mathrm{Li}_{3 x} \mathrm{TiO}_{3}$ (LLTO) attracted lots of attention due to its potentially application in all solid state lithium ion battery. In 1993, Inaguma Yoshiyuki's first cubic perovskite structure LLTO. The grain bulk ionic conductivity can reach $10^{-3} \mathrm{~S} / \mathrm{cm}$, but grain boundary ionic conductivity was 3 orders lower than the grain bulk, so find ways to increase the ionic conductivity at the grain boundary is necessary. Previously people add amorphous phases into LLTO grain boundary to improve its ionic conductivity, some of them works really well.

Sulfide glasses have the highest lithium ion conductivity among all the glassy lithium ion conductor, however, easy to absorb moisture is the main barrier to fabrication and doping into LLTO. This is the reason we choose oxide glass system doping into LLTO grain boundary to enhance the ionic conductivity.

An electrochemical impedance spectroscopy investigation of the composite $\mathrm{LLTO} /\left(0.4 \mathrm{Li}_{2} \mathrm{O}+0.25 \mathrm{~B}_{2} \mathrm{O}_{3}+(0.35-\mathrm{x}) \mathrm{SiO}_{2}+\mathrm{xAl}_{2} \mathrm{O}_{3}\right)$ electrolyte shows that the grain boundary conductivity can be improved by 2 orders of magnitude at room temperature. The two roles of $\mathrm{Al}_{2} \mathrm{O}_{3}$ in this glass system are to prevent the crystallization of $\mathrm{Li}_{2} \mathrm{O}-\mathrm{B}_{2} \mathrm{O}_{3}-\mathrm{SiO}_{2}$ glass and to create lithium ion defects at the LLTO interface. Based on density measurements, the $2 \mathrm{wt} \%$ oxide glass component was found to have the greatest density and so be the optimum amount necessary to fully fill the LLTO grain boundary regions. Excess $\mathrm{Al}_{2} \mathrm{O}_{3}$ in this glass system was found detrimental the ionic conductivity property and will reduce the ion conductivity due to the inherent lithium defects formed in the base LLTO were replaced by aluminum, thus stack the lithium ions penetration. 


\section{Reference:}

[1] Väyrynen, Antti, and Justin Salminen. "Lithium ion battery production." The Journal of Chemical Thermodynamics 46 (2012): 80-85.

[2] Verma, Pallavi, Pascal Maire, and Petr Novák. "A review of the features and analyses of the solid electrolyte interphase in Li-ion batteries." Electrochimica Acta 55.22 (2010): 6332-6341.

[3] Patil, Arun, et al. "Issue and challenges facing rechargeable thin film lithium batteries." Materials research bulletin 43.8 (2008): 1913-1942.

[4] Liao, Cheng-Lung, Chung-Han Wen, and Kuan-Zong Fung. "The stability between perovskite La $2 / 3-x$ Li $3 x \square 1 / 3-2 x$ TiO $3(3 x=0.3)$ electrolyte and LiM m O n (M= Mn, Ni and Co) cathodes." Journal of alloys and compounds 432.1 (2007): L22-L25.

[5] Li, Zhe, et al. "A review of lithium deposition in lithium-ion and lithium metal secondary batteries." Journal of power sources 254 (2014): 168-182.

[6] Ahn, Jun-Ku, Soon-Gil Yoon, and Chang Soo Kim. "Effect of Li0. 5La0. 5TiO3 solid electrolyte films on electrochemical properties of $\mathrm{LiCoO} 2$ thin film cathodes with different rapidthermal annealing conditions." Journal of Vacuum Science \& Technology B 23.5 (2005): 20892094.

[7] Endo, M., et al. "Recent development of carbon materials for Li ion batteries." Carbon 38.2 (2000): 183-197.

[8] Väyrynen, Antti, and Justin Salminen. "Lithium ion battery production." The Journal of Chemical Thermodynamics 46 (2012): 80-85.

[9] Trevey, James, et al. "Glass-ceramic Li 2 S-P 2 S 5 electrolytes prepared by a single step ball billing process and their application for all-solid-state lithium-ion batteries." Electrochemistry communications 11.9 (2009): 1830-1833.

[10] Tatsumisago, Masahiro, Fuminori Mizuno, and Akitoshi Hayashi. "All-solid-state lithium secondary batteries using sulfide-based glass-ceramic electrolytes." Journal of power sources 159.1 (2006): 193-199. 
[11] Minami, Tsutomu, Akitoshi Hayashi, and Masahiro Tatsumisago. "Recent progress of glass and glass-ceramics as solid electrolytes for lithium secondary batteries." Solid State Ionics 177.26 (2006): 2715-2720.

[12] Minami, Keiichi, et al. "Structure and properties of Li 2 S-P 2 S 5-P 2 S 3 glass and glassceramic electrolytes." Journal of Power Sources 189.1 (2009): 651-654.

[13] Nishio, Yusuke, et al. "All-solid-state lithium secondary batteries using nanocomposites of NiS electrode/Li 2 S-P 2 S 5 electrolyte prepared via mechanochemical reaction." Journal of Power Sources 189.1 (2009): 629-632.

[14] Hayashi, Akitoshi, et al. "Novel technique to form electrode-electrolyte nanointerface in allsolid-state rechargeable lithium batteries." Electrochemistry Communications 10.12 (2008): 18601863.

[15] Hayashi, Akitoshi, Ryoji Ohtsubo, and Masahiro Tatsumisago. "Electrochemical performance of all-solid-state lithium batteries with mechanochemically activated $\mathrm{Li} 2 \mathrm{~S}-\mathrm{Cu}$ composite electrodes." Solid State Ionics 179.27 (2008): 1702-1705.

[16] Nagao, Motohiro, et al. "Characterization of all-solid-state lithium secondary batteries using Cu x Mo 6 S 8- y electrode and Li 2 S-P 2 S 5 solid electrolyte." Journal of Power Sources 189.1 (2009): 672-675.

[17] Fu, Jie. "Superionic conductivity of glass-ceramics in the system $\mathrm{Li} 2 \mathrm{O} \cdot \mathrm{Al} 2 \mathrm{O} 3 \cdot \mathrm{TiO}$ 2 • P 2 O 5." Solid State Ionics 96.3 (1997): 195-200.

[18] Fu, Jie. "Fast $\mathrm{Li}+$ ion conducting glass-ceramics in the system $\mathrm{Li} 2 \mathrm{O}-\mathrm{Al} 2 \mathrm{O} 3-\mathrm{GeO} 2-\mathrm{P} 2$ O 5." Solid State Ionics 104.3 (1997): 191-194.

[19] Chowdari, B. V. R., GV Subba Rao, and G. Y. H. Lee. "XPS and ionic conductivity studies on Li 2 O-Al 2 O 3-(TiO 2 or GeO 2)-P 2 O 5 glass-ceramics." Solid State Ionics 136 (2000): 1067-1075.

[20] Xu, Xiaoxiong, et al. "Lithium ion conductive glass ceramics in the system Li 1.4 Al 0.4 (Ge 1-x Ti x) 1.6 (PO 4) 3 (x=0-1.0)." Solid State Ionics 171.3 (2004): 207-213. 
[21] Xu, Xiaoxiong, et al. "High lithium ion conductivity glass-ceramics in Li 2 O-Al 2 O 3-TiO

2-P 2 O 5 from nanoscaled glassy powders by mechanical milling." Solid State Ionics 177.26 (2006): 2611-2615.

[22] Thokchom, Joykumar S., and Binod Kumar. "Microstructural effects on the superionic conductivity of a lithiated glass-ceramic." Solid State Ionics 177.7 (2006): 727-732.

[23] Tang, Mengxuan, et al. "Lithium-Stable High Lithium Ion Conducting Li1. 4Al0. 4Ge0. 2 Ti1.

4 (PO4) 3 Solid Electrolyte." Journal of The Electrochemical Society 163.9 (2016): A1822-A1828.

[24] Inaguma, Yoshiyuki, et al. "High ionic conductivity in lithium lanthanum titanate." Solid State Communications 86.10 (1993): 689-693.

[25] Várez, A., et al. "Microstructural Study of La 0.5 Li 0.5 TiO 3." Journal of Solid State Chemistry 118.1 (1995): 78-83.

[26] Fourquet, J. L., H. Duroy, and M. P. Crosnier-Lopez. "Structural and Microstructural Studies of the Series La 2/3-x Li 3 x $\square$ 1/3-2x TiO 3." Journal of Solid State Chemistry 127.2 (1996): 283-294.

[27] Inaguma, Yoshiyuki, et al. "Crystal structure of a lithium ion-conducting perovskite La 2/3x Li 3x TiO 3 (x= 0.05)." Journal of Solid State Chemistry 166.1 (2002): 67-72.

[28] Varez, A., et al. "Structural modifications induced by high-temperature quenching treatments in the fast ion conductor Li0. 18La0. 61TiO3: a neutron diffraction study." Chemistry of materials 15.24 (2003): 4637-4641.

[29] Sanz, Jesus, et al. "Structural changes produced during heating of the fast ion conductor Li 0.18 La 0.61 TiO 3. A neutron diffraction study." Journal of Solid State Chemistry 177.4 (2004): 1157-1164.

[30] Mozhegorov, A. A., et al. "Structure and the electronic and magnetic properties of LaTiO3." Physics of the Solid State 50.9 (2008): 1795-1798.

[31] Eitel, Manfred, and J. E. Greedan. "A high resolution neutron diffraction study of the perovskite LaTiO 3." Journal of the Less Common Metals 116.1 (1986): 95-104. 
[32] Sommariva, Marco, and Michele Catti. "Neutron diffraction study of quenched Li0. 3La0. 567TiO3 lithium ion conducting perovskite." Chemistry of materials 18.9 (2006): 2411-2417.

[33] "X-ray photoelectron spectroscopic and secondary ion mass spectroscopic examinations of metallic-lithium-activated donor doping process on $\mathrm{La} 0$. 56 $\mathrm{Li} 0$. 33TiO3 surface at room temperature." Journal of applied physics 100, no. 5 (2006): 05610-2.

[34] Catti, Michele. "Ion mobility pathways of the Li+ conductor Li0. 125La0. 625TiO3 by ab initio simulations." The Journal of Physical Chemistry C 112.29 (2008): 11068-11074.

[35] Catti, Michele. "First-Principles Modeling of Lithium Ordering in the LLTO (Li x La2/3x/3TiO3) Superionic Conductor." Chemistry of materials 19.16 (2007): 3963-3972.

[36] Moriwake, Hiroki, et al. "Domain boundaries and their influence on Li migration in solidstate electrolyte (La, Li) TiO 3." Journal of Power Sources 276 (2015): 203-207.

[37] Gao, Xiang, et al. "Lithium atom and A-site vacancy distributions in lanthanum lithium titanate." Chemistry of Materials 25.9 (2013): 1607-1614.

[38] Yashima, Masatomo, et al. "Crystal structure and diffusion path in the fast lithium-ion conductor La0. 62Li0. 16TiO3." Journal of the American Chemical Society 127.10 (2005): 34913495 .

[39] Kim, Dae-Hee, et al. "Lithium ion migration pathways in Li 3x La 2/3-x $\square$ 1/3-2x TiO 3." Ceramics International 38 (2012): S467-S470.

[40] Mazza, Daniele, et al. "Modeling Li-ion conductivity in fast ionic conductor $\mathrm{La} 2 / 3-\mathrm{x} \mathrm{Li} \mathrm{3x}$ TiO 3." Solid State Ionics 149.1 (2002): 81-88.

[41] Catti, Michele. "Short-range order and Li+ ion diffusion mechanisms in Li $5 \mathrm{La} 9 \square 2$ (TiO 3) 16 (LLTO)." Solid State Ionics 183.1 (2011): 1-6.

[42] Mei, Ao, et al. "Enhanced ionic transport in lithium lanthanum titanium oxide solid state electrolyte by introducing silica." Solid State Ionics 179.39 (2008): 2255-2259.

[43] Wada, H., et al. "Preparation and ionic conductivity of new B2S3-Li2S-LiI glasses." Materials research bulletin 18.2 (1983): 189-193. 
[44] Pradel, Annie, Thierry Pagnier, and Michel Ribes. "Effect of rapid quenching on electrical properties of lithium conductive glasses." Solid State Ionics 17.2 (1985): 147-154.

[45] Kennedy, John H., and Zhengming Zhang. "Improved stability for the SiS 2-P 2 S 5-Li 2 SLiI glass system." Solid State Ionics 28 (1988): 726-728.

[46] Pradel, A., and M. Ribes. "Lithium chalcogenide conductive glasses." Materials chemistry and physics 23.1 (1989): 121-142.

[47] Kennedy, J. H. "Ionically conductive glasses based on SiS 2." Materials chemistry and physics 23.1 (1989): 29-50.

[48] Zhang, Zhengming, and John H. Kennedy. "Synthesis and characterization of the B2S3 • Li2S, the P2S5 - Li2S and the B2S3 - P2S5 • Li2S glass systems." Solid State Ionics 38.3-4 (1990): 217-224.

[49] Tatsumisago, Masahiro, et al. "Superionic conduction in rapidly quenched Li2S-SiS2-Li3PO4 glasses." Nippon seramikkusu kyokai gakujutsu ronbunshi 101.11 (1993): 1315-1317.

[50] Aotani, Noboru, et al. "Synthesis and electrochemical properties of lithium ion conductive glass, Li 3 PO 4 Li 2 S SiS 2." Solid State Ionics 68.1 (1994): 35-39.

[51] Hirai, Koichi, Masahiro Tatsumisago, and Tsutomu Minami. "Thermal and electrical properties of rapidly quenched glasses in the systems $\mathrm{Li} 2 \mathrm{~S} \cdot \mathrm{SiS} 2 \cdot \mathrm{Li}$ x MO y $(\mathrm{Li} \times \mathrm{MO}$ y= Li 4 SiO 4, Li 2 SO 4)." Solid State Ionics 78.3 (1995): 269-273.

[52] Tatsumisago, Masahiro, et al. "Structure and properties of lithium ion conducting oxysulfide glasses prepared by rapid quenching." Solid State Ionics 86 (1996): 487-490.

[53] Tatsumisago, M., et al. "Preparation and characterisation of superionic Li2S-SiS2-Li4GeO4 glasses." Physics and chemistry of glasses 38.2 (1997): 63-65.

[54] Minami, Tsutomu, Akitoshi Hayashi, and Masahiro Tatsumisago. "Preparation and characterization of lithium ion-conducting oxysulfide glasses." Solid State Ionics 136 (2000): 1015-1023. 
[55] Minami, Tsutomu, Akitoshi Hayashi, and Masahiro Tatsumisago. "Recent progress of glass and glass-ceramics as solid electrolytes for lithium secondary batteries." Solid State Ionics 177.26 (2006): 2715-2720.

[56] Hayashi, Akitoshi, et al. "Thermal and electrical properties of rapidly quenched Li 2 S-SiS 2Li 2 OP 2 O 5 oxysulfide glasses." Solid state ionics 113 (1998): 733-738.

[57] K. Otto, Phys. Chem. Glasses 7 [ I ] (1966) 29.

[58] Kim, Young Han, et al. "Effect of $\mathrm{SiO} 2 / \mathrm{B} 2 \mathrm{O} 3$ ratio on Li ion conductivity of a Li2O-B2O3SiO2 glass electrolyte." Journal of Ceramic Processing Research 13 (2012): 37-41.

[59] Kim, Chul Eui, et al. "Fabrication of a high lithium ion conducting lithium borosilicate glass." Journal of Non-Crystalline Solids 357.15 (2011): 2863-2867.

[60] Tatsumisago, Masahiro, et al. "Ionic conductivity of rapidly quenched glasses with high concentration of lithium ions." Journal of Non-Crystalline Solids 95 (1987): 857-864.

[61] Rogez, J., et al. "Determination of the crystallization enthalpies of lithium ion conducting alumino-silicate glasses." Journal of non-crystalline solids 262.1 (2000): 177-182.

[62] Hsieh, C. H., and H. Jain. "Influence of network-forming cations on ionic conduction in sodium silicate glasses." Journal of non-crystalline solids 183.1 (1995): 1-11.

[63] Deng, Yuan, et al. "The preparation and conductivity properties of Li $0.5 \mathrm{La} 0.5 \mathrm{TiO}$ 3/inactive second phase composites." Journal of Alloys and Compounds 472.1 (2009): 456-460.

[64] Maier, Joachim. "Ionic conduction in space charge regions." Progress in solid state chemistry 23.3 (1995): 171-263.

[65] Kim, Dong Hun, et al. "Li loss during the growth of ( $\mathrm{Li}, \mathrm{La}) \mathrm{TiO} 3$ thin films by pulsed laser deposition." Journal of Crystal Growth 372 (2013): 9-14.

[66] Vijayakumar, Murugesan, et al. "Synthesis of Fine Powders of Li3 x La2/3-x TiO3 Perovskite by a Polymerizable Precursor Method." Chemistry of Materials 16.14 (2004): 2719-2724.

[67] Maqueda, Oscar, et al. "Structural, microstructural and transport properties study of lanthanum lithium titanium perovskite thin films grown by Pulsed Laser Deposition." Thin Solid Films 516.8 (2008): 1651-1655. 
[68] Geng, H. X., et al. "Investigation of structure and electrical properties of Li 0.5 La 0.5 TiO 3 ceramics via microwave sintering." Journal of Alloys and Compounds 481.1 (2009): 555-558.

[69] Mei, Ao, et al. "Lithium lanthanum titanium oxide solid-state electrolyte by spark plasma sintering." Journal of Alloys and Compounds 486.1 (2009): 871-875.

[70] Wolfenstine, J., et al. "Hot-pressed Li 0.33 La 0.57 TiO 3." Journal of Power Sources 195.13 (2010): 4124-4128.

[71] Ban, Chang Wan, and Gyeong Man Choi. "The effect of sintering on the grain boundary conductivity of lithium lanthanum titanates." Solid State Ionics 140.3 (2001): 285-292.

[72] Mei, Ao, et al. "Role of amorphous boundary layer in enhancing ionic conductivity of lithiumlanthanum-titanate electrolyte." Electrochimica Acta 55.8 (2010): 2958-2963.

[73] Zhang, Hui, et al. "On the La 2/3-xLi 3 xTiO 3/Al 2 O 3 composite solid-electrolyte for Liion conduction." Journal of Alloys and Compounds 577 (2013): 57-63.

[74] Abrahams, Isaac, and Emina Hadzifejzovic. "Lithium ion conductivity and thermal behaviour of glasses and crystallised glasses in the system Li 2 O-Al 2 O 3-TiO 2-P 2 O 5." Solid State Ionics 134.3 (2000): 249-257.

[75] Fu, Jie. "Fast $\mathrm{Li}+$ ion conducting glass-ceramics in the system $\mathrm{Li} 2 \mathrm{O}-\mathrm{Al} 2 \mathrm{O}$ 3-GeO 2-P 2 O 5." Solid State Ionics 104.3 (1997): 191-194. 